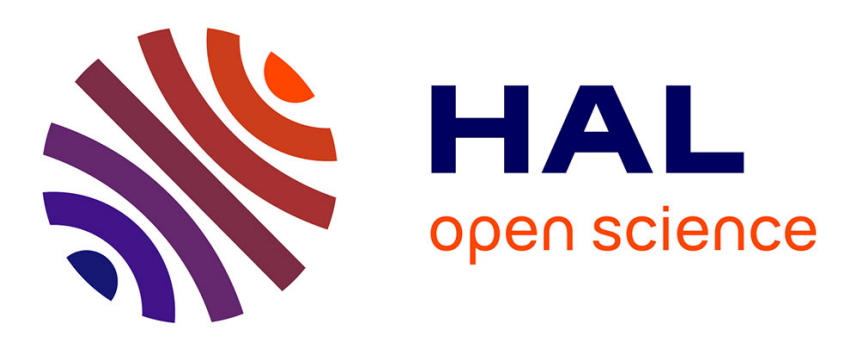

\title{
Non-leaky surface acoustic waves in the passbands of one-dimensional phononic crystals
}

\author{
A.N. Darinskii, A.L. Shuvalov
}

\section{To cite this version:}

A.N. Darinskii, A.L. Shuvalov. Non-leaky surface acoustic waves in the passbands of one-dimensional phononic crystals. Ultrasonics, 2019, 98, pp.108-118. 10.1016/j.ultras.2019.06.002 . hal-02396055

\section{HAL Id: hal-02396055 \\ https://hal.science/hal-02396055}

Submitted on 15 Dec 2020

HAL is a multi-disciplinary open access archive for the deposit and dissemination of scientific research documents, whether they are published or not. The documents may come from teaching and research institutions in France or abroad, or from public or private research centers.
L'archive ouverte pluridisciplinaire HAL, est destinée au dépôt et à la diffusion de documents scientifiques de niveau recherche, publiés ou non, émanant des établissements d'enseignement et de recherche français ou étrangers, des laboratoires publics ou privés. 


\title{
Non-leaky surface acoustic waves in the passbands of one-dimensional phononic
}

\author{
crystals
}

\author{
A.N. Darinskiia ${ }^{a, b)}$, A.L. Shuvalov ${ }^{c)}$ \\ a) Shubnikov Institute of Crystallography \\ FSRC "Crystallography and Photonics", \\ Russian Academy of Sciences, Leninskii pr. 59, Moscow 119333, Russia \\ b) National University of Science and Technology "MISIS", \\ Leninsky pr. 4, Moscow 119049, Russia \\ c)Univ. Bordeaux, CNRS, UMR 5295, 33405 Talence, France
}

\begin{abstract}
The paper theoretically investigates the occurrence of non-leaky surface acoustic waves (SAWs) in the passbands of the Floquet-Bloch spectra of half-infinite one-dimensional phononic crystals. The phononic crystal is represented by a periodic structure of perfectly bonded anisotropic elastic layers. The traction-free boundary plane truncates the phononic crystal at the edge of a period. For the general case of unrestricted anisotropy of the constitutive layers, it is shown that
\end{abstract}


if the passband allows only two partial bulk modes, then the nonleaky SAW must simultaneously satisfy three real equations imposed on problem parameters such as the SAW frequency and tangential wavenumber, the angle of its propagation direction along the given boundary plane, and the characteristics of the medium. If there are four bulk modes in the passband, then the non-leaky SAW must satisfy at least five real equations. In the case where the layers possess a common plane of crystallographic symmetry which is either parallel to the layer interfaces or perpendicular to the direction of propagation, the number of real equations conditioning the occurrence of non-leaky SAWs in the passbands with two and with four bulk modes reduces to two and three, respectively. If the sagittal plane is a plane of symmetry, then the sagittally polarized non-leaky passband SAW must satisfy three real equations; however, if this sagittal symmetry plane coexists with another plane of symmetry which is either parallel to the layer interfaces or perpendicular to the direction of propagation, then the existence of non-leaky passband SAW requires the fulfillment of two conditions only. In particular, this is always the case when the constitutive layers are elastically isotropic. The general conclusions are illustrated by numerical examples. 
Keywords: one-dimensional phononic crystal, supperlattice, surface acoustic wave, passband 


\section{Introduction}

Surface acoustic waves (SAWs) on half-infinite periodic superlattices, otherwise termed one-dimensional (1D) phononic crystals, continue to attract researchers' attention due to the wide use of such waves for various applications such as non-destructive evaluation of layered composites [1], signal processing [2], etc. One of the most heavily studied subjects is the propagation of shear horizontally polarized SAWs modes on solid non-piezoelectric and piezoelectric 1D phononic crystals [3]-[9]. Sagittally polarized two-partial SAWs [11]-[15] and fully coupled three-partial SAWs [16]-[18] were also investigated. In addition, the SAW propagation on solid-fluid periodic [19, 20] and Fibonacci [21] structures were studied both analytically and numerically. Comprehensive discussions of various properties of SAWs and of bulk acoustic waves in 1D phononic crystals as well as in 2D and 3D phononic crystals can be found in [22]-[25].

The Floquet-Bloch partial modes in a 1D periodic structure may have the amplitudes which either stay constant from one period edge to another or increase/decrease into the depth. By analogy with waves in homogeneous media, these modes may be referred to as either bulk or inhomogeneous ones, respectively. Their frequency $\omega$ and the tangential wavenumber $k$ belong to either passband or stopband areas on the real-valued $(\omega, k)$ plane. Usually SAWs occur in the so-called full stopbands which do not admit any bulk partial modes, i.e. all the partial modes there are inhomogeneous. In Ref. [26] we analyzed the existence of such SAWs in half-infinite 1D phononic crystals assembled from elastic layers of unrestricted anisotropy. According to [26], not more than three SAW branches $\omega(k)$ can exist in a full stopband 
of a phononic crystal with an arbitrary periodic composition of layers. It was also shown in [26] that if the layers are arranged in a way which makes the period to be symmetric with respect to its midplane, then any full stopband cannot admit more than one SAW branch.

Half-infinite 1D phononic crystals also support leaky waves, which are usually characterized by a complex frequency $\omega$ and a real wavenumber $k$ $[14,16,17,19]$. The values of the real part of the leaky-wave frequency and of the corresponding real wavenumber fall in the passbands, i.e., in the areas of $(\omega, k)$ plane which admit bulk modes. Due to the coupling between surface and bulk modes, the bulk-wave reflection and transmission, when occurring in the vicinity of the leaky-wave branch, are characterized by the resonant features, which overlap the resonances of the interference type brought in by the periodicity of the phononic crystal and which become sharper with decreasing of the imaginary part of the leaky-wave frequency, see e.g., [16]. It appears that there may exist special orientations in a half-infinite phononic crystal, for which the leaky wave transforms into a non-leaky SAW with a real frequency lying in the passband. An example of such passband SAWs propagating on the boundary of GaAs/AlAs periodically layered structure was numerically found in Ref. [17].

Note aside that, to some extent, the SAWs occurring in the full stopbands and the SAWs occurring in the passbands resemble, respectively, the so-called subsonic SAWs [27, 28] and the supersonic SAWs [29]-[33] propagating on homogeneous substrates.

The present paper analyzes the existence conditions for the passband SAWs on half-infnite 1D phononic crystals. It could be surmised that at most 
two conditions stipulate the existence of the passband SAW. Indeed, since the surface impedance $\hat{\mathbf{Z}}$ of a half-infinite phononic crystal is a non-Hermitian matrix in passbands and hence its determinant therein is complex-valued, one could argue that the real and imaginary parts of the dispersion equation $\operatorname{det}(\hat{\mathbf{Z}})=0$ impose two conditions on the parameters of the problem, thus defining the real frequency and real wavenumber $k$ as functions, e.g, of an orientation angle. However, we will show that this reasoning is faulty and that in fact the existence of passband SAWs is generally conditioned by more than two relations. Meeting just two conditions is sufficient only in the case of certain symmetric orientations and in the phononic crystals composed of isotropic layers. Note also that the problem of the passband SAWs is not addressed by the theory [26], which considers SAWs in the full stopbands and therefore makes essential use of the fact that all partial modes in a full stopband are inhomogeneous ones.

The paper is organized as follows. The background of the problem is presented in section 2 where we remind the basic principles of the transfermatrix formalism for waves in periodic media. Section 3 contains the analysis of the existence conditions for the passband SAWs in the general case of unrestricted anisotropy of the constitutive layers and of their arbitrary ordering within a period. Effect of the crystallographic symmetry of layers on the occurrence of passband SAWs is considered in section 4. Section 5 examines existence of passband SAWs in the case of particular symmetric ordering of layers within a period. Numerical examples are given in section 6 . The conclusions are provided in section 7. 


\section{Transfer-matrix formalism}

Consider a structure of solid non-piezoelectric and non-viscous homogeneous layers. Each $j$-th layer has density $\rho^{(j)}$ and elastic moduli $c_{i k l m}^{(j)}$. Timeharmonic acoustic wave with a frequency $\omega$ propagating in a $j$-th layer satisfies the equation

$$
\frac{\partial \sigma_{i k}}{\partial x_{k}}=-\rho^{(j)} \omega^{2} u_{i}
$$

where

$$
\sigma_{i k}=c_{i k l m}^{(j)} \partial u_{l} / \partial x_{m}, \quad i, k, l, m=1,2,3,
$$

are the components of the mechanical stress tensor $\hat{\sigma}, u_{i}$ are the components of the displacement vector $\mathbf{u}$, and $x_{i}$ are the components of the radius-vector $\mathbf{r}$. Denote by $\mathbf{n}$ and $\mathbf{m}$ the unit vectors which are, respectively, perpendicular and parallel to the layer interfaces. We seek the displacement in the form

$$
\mathbf{u}(\mathbf{r}, t)=\mathbf{a}(y) \mathrm{e}^{i[k(\mathbf{m r})-\omega t]},
$$

where $y=\mathbf{n r}$ and $k$ is the tangential wavenumber. Let us introduce a column vector of displacement and traction amplitudes $\xi(y)=(\mathbf{a}(y), \mathbf{l}(y))^{t}$, where $\mathbf{l}=i k^{-1} \hat{\sigma} \mathbf{n}$ and the symbol ${ }^{t}$ stands for transposition. Eqs. (1) and (2) applied to the displacement field (3) in a $j$-th layer can be combined into the form [34]

$$
\frac{1}{i k} \frac{d \xi}{d y}=\hat{\mathbf{N}}_{j} \xi
$$

with the $6 \times 6$ Stroh matrix

$$
\hat{\mathbf{N}}_{j}=-\left(\begin{array}{cc}
(n n)^{-1}(n m) & (n n)^{-1} \\
(m n)(n n)^{-1}(n m)-(m m)+\rho^{(j)}(\omega / k)^{2} \hat{\mathbf{I}} & (m n)(n n)^{-1}
\end{array}\right)
$$


in which $\hat{\mathbf{I}}$ is the $3 \times 3$ unit matrix and the $3 \times 3$ matrices $(a b)$ have the components $(a b)_{k l}^{(j)}=a_{i} c_{i k l m}^{(j)} b_{m}$ with $\mathbf{a}, \mathbf{b}=\mathbf{n}$ or $\mathbf{m}$. The matrix $\hat{\mathbf{N}}_{j}$ possesses the property

$$
\hat{\mathbf{N}}_{j}=\hat{\mathbf{T}} \hat{\mathbf{N}}_{j}^{t} \hat{\mathbf{T}}
$$

where

$$
\hat{\mathbf{T}}=\left(\begin{array}{cc}
\hat{\mathbf{O}} & \hat{\mathbf{I}} \\
\hat{\mathbf{I}} & \hat{\mathbf{O}}
\end{array}\right)
$$

and $\hat{\mathbf{O}}$ is the $3 \times 3$ zero matrix.

Let the layered structure consist of periodically repeated stacks of $n$ perfectly bonded layers with thicknesses $h_{j}, j=1, \ldots, n$. Since the displacement and traction are continuous at all internal interfaces, the transfer matrix through a period is [15]

$$
\hat{\mathbf{M}}=\hat{\mathbf{M}}_{n} \hat{\mathbf{M}}_{n-1} \cdots \hat{\mathbf{M}}_{1}=\mathrm{e}^{i k h_{n} \hat{\mathbf{N}}_{n}} \mathrm{e}^{i k h_{n-1} \hat{\mathbf{N}}_{n-1}} \cdots \mathrm{e}^{i k h_{1} \hat{\mathbf{N}}_{1}}
$$

where $\hat{\mathbf{M}}_{j}=\exp \left(i k h_{j} \hat{\mathbf{N}}_{j}\right)$ is the transfer matrix through a $j$-th layer. The fundamental role in the description of wave propagation through a periodic structure is played by the eigenvalue problem of $\hat{\mathbf{M}}$,

$$
\hat{\mathbf{M}} \zeta_{\alpha}=\gamma_{\alpha} \zeta_{\alpha}, \quad \alpha=1, \ldots, 6
$$

where the eigenvectors $\zeta_{\alpha}=\left(\mathbf{A}_{\alpha}, \mathbf{L}_{\alpha}\right)^{t}$ are six-component vector columns, whose components $\mathbf{A}_{\alpha}$ and $\mathbf{L}_{\alpha}$ have the meaning of, respectively, the displacement and traction. The displacement-traction field $\xi_{\alpha}(y)$ generated by the $\alpha$-th eigenvector at some point $y=\tilde{y}+p \sum_{j=1}^{n} h_{j}+\sum_{j=q}^{n} h_{j}$ inside $q^{\text {th }}$ layer of the $p^{\text {th }}$ period,

$$
\xi_{\alpha}(y)=\gamma_{\alpha}^{p-1} \exp \left(i \tilde{y} \hat{\mathbf{N}}_{q}\right) \prod_{j=1}^{q-1} \hat{\mathbf{M}}_{j} \zeta_{\alpha}
$$


is called the $\alpha$-th partial mode.

In view of the symmetry (6) of the matrices $\hat{\mathbf{N}}_{j}$, the transfer matrix $\hat{\mathbf{M}}$ (8) taken at real $\omega$ and $k$ obeys the equality $[15,35]$

$$
\hat{\mathbf{M}}^{-1}=\hat{\mathbf{T}} \hat{\mathbf{M}}^{\dagger} \hat{\mathbf{T}}
$$

where the superscript $\nmid$ stands for the Hermitian transpose. Applying the property (11) to the eigenproblem (9) yields the identity $\left(\gamma_{\alpha}^{*} \gamma_{\beta}-1\right) \zeta_{\alpha}^{\dagger} \hat{\mathbf{T}} \zeta_{\beta}=$ 0 for any $\alpha, \beta=1, \ldots, 6$, where $*$ means complex conjugate. Given that all $\gamma_{\alpha}$ are distinct, it follows that the eigenvalues and eigenvectors of $\hat{\mathbf{M}}$ come out in pairs $(\alpha, \alpha+3)$, which satisfy either the equality

$$
\left|\gamma_{\alpha}\right|=\left|\gamma_{\alpha+3}\right|=1
$$

and the orthonormalization condition

$$
\zeta_{\alpha}^{\dagger} \hat{\mathbf{T}} \zeta_{\beta}=\delta_{\alpha, \beta}, \zeta_{\alpha+3}^{\dagger} \hat{\mathbf{T}} \zeta_{\beta}=-\delta_{\alpha+3, \beta}, \quad \beta=1, \ldots, 6
$$

or the equality

$$
\gamma_{\alpha}=\frac{1}{\gamma_{\alpha+3}^{*}} \text { for }\left|\gamma_{\alpha}\right| \neq 1,
$$

and the orthonormalization condition

$$
\zeta_{\alpha}^{\dagger} \hat{\mathbf{T}} \zeta_{\beta}=\delta_{\alpha+3, \beta}, \quad \beta=1, \ldots, 6
$$

where $\delta_{\alpha, \beta}$ is the Kronecker symbol and $\alpha$ is fixed among 1,2,3.

The moduli of the eigenvalues $\gamma_{\alpha}$ of $\hat{\mathbf{M}}$ determine the Floquet-Bloch spectrum, i.e. the passband and stopband areas on the real-valued $(\omega, k)$ plane. The area where $\left|\gamma_{\alpha}\right|=\left|\gamma_{\alpha+3}\right|=1$ is the passband of the partial modes $\alpha$ and $\alpha+3$. According to the defintion (10), these modes $\xi_{\alpha}(y)$ and $\xi_{\alpha+3}(y)$ retain 
a constant absolute value on the period edges and so they can be called bulk modes. The area where $\left|\gamma_{\alpha}\right|=1 /\left|\gamma_{\alpha+3}\right| \neq 1$ is the stopband of the partial modes $\alpha$ and $\alpha+3$, one decreasing and the other increasing as number of periods grows away from the reference level specified. These modes with $\left|\gamma_{\alpha}\right|<1$ and $\left|\gamma_{\alpha}\right|>1$ will be called inhomogeneous decaying and increasing ones, respectively. Note that our assumption of distinct $\gamma_{\alpha}$ implies barring the passband and stiopband edges.

The presence of the opposite signs in (13) is formally related to the fact that the matrix $\hat{\mathbf{T}}(7)$ is a non-singular symmetric matrix with a zero signature - zero difference between the number of positive and negative diagonal entries - and that any its conjunctive transformation $\hat{\mathbf{B}}^{\dagger} \hat{\mathbf{T}} \hat{\mathbf{B}}$ by a non-singular matrix $\hat{\mathbf{B}}$ retains the signature, hence a conjunctive transformation of $\hat{\mathbf{T}}$ by the matrix with columns being the eigenvectors $\zeta_{\alpha}$ leads to the opposite signs in (13), see $[35,36]$. This feature may also be understood from the physical viewpoint. The time-averaged energy flow $P_{n, \alpha}$ of the mode $\alpha$ along the normal $\mathbf{n}$ to the layer interfaces is equal to $P_{n, \alpha}=-\omega k \zeta_{\alpha}^{\dagger} \hat{\mathbf{T}} \zeta_{\alpha} / 4$. The two bulk modes satisfying (12) must carry normal energy flows in the mutually opposite directions, hence their self-products $\zeta_{\alpha}^{\dagger} \hat{\mathbf{T}} \zeta_{\alpha}$ and $\zeta_{\alpha+3}^{\dagger} \hat{\mathbf{T}} \zeta_{\alpha+3}$ must be of opposite signs. In the following we will assume that the phononic crystal occupies the half-space $y=(\mathbf{n r})>0$, and so the positive and negative self-product in (13) will correspond to the incident and reflected modes, respectively.

Note also that Eqs. (13) and (15) can be used to demonstrate that (i) the vectors $\mathbf{L}_{\alpha}$ corresponding to the inhomogeneous modes cannot be parallel with either of $\mathbf{L}_{i}$ and $\mathbf{L}_{r}$, (ii) a pair of $\mathbf{L}_{\alpha}$, which does not consist of $\alpha$-th 
and $(\alpha+3)$-th vectors, cannot be coplanar with either of $\mathbf{L}_{i}$ and $\mathbf{L}_{r}$. These observations appear helpful for the subsequent considerations in $\S 3$.

Provided that the eigenvectors of $\hat{\mathbf{M}}$ are normalized according to Eqs. $(13),(15)$ and two of them are associated with the bulk modes, the completeness relation reads as

$$
\zeta_{i} \otimes \hat{\mathbf{T}} \zeta_{i}^{*}-\zeta_{r} \otimes \hat{\mathbf{T}} \zeta_{r}^{*}+\sum_{\alpha=2}^{3}\left(\zeta_{\alpha} \otimes \hat{\mathbf{T}} \zeta_{\alpha+3}^{*}+\zeta_{\alpha+3} \otimes \hat{\mathbf{T}} \zeta_{\alpha}^{*}\right)=\left(\begin{array}{cc}
\hat{\mathbf{I}} & \hat{\mathbf{O}} \\
\hat{\mathbf{O}} & \hat{\mathbf{I}}
\end{array}\right)
$$

where the symbol $\otimes$ stands for the dyadic product, the subscripts $i$ and $r$ label the bulk incident and reflected modes, respectively, and the subscripts $\alpha=2,3,5,6$ correspond to the inhomogeneous modes. If there are two pairs of bulk modes in the passband, then the completeness relation acquires the form

$$
\sum_{\alpha=i 1, i 2} \zeta_{\alpha} \otimes \hat{\mathbf{T}} \zeta_{\alpha}^{*}-\sum_{\alpha=r 1, r 2} \zeta_{\alpha} \otimes \hat{\mathbf{T}} \zeta_{\alpha}^{*}+\zeta_{3} \otimes \hat{\mathbf{T}} \zeta_{6}^{*}+\zeta_{6} \otimes \hat{\mathbf{T}} \zeta_{3}^{*}=\left(\begin{array}{cc}
\hat{\mathbf{I}} & \hat{\mathbf{O}} \\
\hat{\mathbf{O}} & \hat{\mathbf{I}}
\end{array}\right),
$$

where the subscripts $i 1, i 2$ and $r 1, r 2$ correspond to the bulk incident and reflected modes, respectively, and the indices 3, 6 correspond to the inhomogeneous modes. For the future use, let us write out explicitly the identities which are expressed by the left $3 \times 3$ off-diagonal block of Eqs. (16) and (17), namely,

$$
\mathbf{L}_{i} \otimes \mathbf{L}_{i}^{*}-\mathbf{L}_{r} \otimes \mathbf{L}_{r}^{*}+\sum_{\alpha=2}^{3}\left(\mathbf{L}_{\alpha} \otimes \mathbf{L}_{\alpha+3}^{*}+\mathbf{L}_{\alpha+3} \otimes \mathbf{L}_{\alpha}^{*}\right)=\hat{\mathbf{O}}
$$

and

$$
\sum_{\alpha=i 1, i 2} \mathbf{L}_{\alpha} \otimes \mathbf{L}_{\alpha}^{*}-\sum_{\alpha=r 1, r 2} \mathbf{L}_{\alpha} \otimes \mathbf{L}_{\alpha}^{*}+\mathbf{L}_{3} \otimes \mathbf{L}_{6}^{*}+\mathbf{L}_{6} \otimes \mathbf{L}_{3}^{*}=\hat{\mathbf{O}} .
$$




\section{Passband SAWs on phononic crystals of ar- bitrary anisotropy and ordering}

From now on we consider a periodically layered half-space $\mathbf{n r}>0$, whose boundary plane $y=0$ coinciding with a period edge is free of traction so that $\hat{\sigma} \mathbf{n}=\mathbf{0}$. The present section is concerned with the general case of unrestricted anisotropy of the constitutive layers and of their arbitrary arrangement in a period. Certain particular cases of the layer crystallographic and arrangement symmetry are discussed $\S \S 4$ and 5 .

\subsection{Preliminary considerations}

Consider a passband where there is one pair of bulk modes $\alpha=i$ and $\alpha=r$ and two pairs of inhomogeneous modes $\alpha=2,3,5,6$, among which the two decaying modes will be identified by the indices $\alpha=2,3$. We start from leaky waves. The leaky wave $\xi_{\text {leaky }}$ is sought as a linear combination $\xi_{\text {leaky }}=$ $\sum_{\alpha=r, 2,3} b_{\alpha} \xi_{\alpha}(y)$ of the partial modes $\alpha=r, 2,3$, where $\xi_{\alpha}(y)$ is given by Eq. (10). By (10), the displacement-traction field of the mode $\alpha$ at the surface $y=0$ is described by the eigenvector $\zeta_{\alpha}$ of the matrix $\hat{\mathbf{M}}$. Thus the partial amplitudes $b_{\alpha}$ are to be found from the traction-free boundary condition

$$
\sum_{\alpha=r, 2,3} b_{\alpha} \mathbf{L}_{\alpha}=\mathbf{0} .
$$

The condition of existence of non-trivial solutions $b_{\alpha}$ of (20) provides the dispersion equation in the form

$$
\left\|\mathbf{L}_{r} \mathbf{L}_{2} \mathbf{L}_{3}\right\|=0
$$


where $\|\cdot\|$ hereafter denotes mixed product of the three enclosed vectors which is identically equal to the determinant of the matrix composed of components of these vectors

Yet another form of the dispersion equation is

$$
\operatorname{det}(\hat{\mathbf{Z}})=0
$$

where $\hat{\mathbf{Z}}$ is the surface impedance of the phononic crystal defined by the relation $\mathbf{L}_{\alpha}=-i \hat{\mathbf{Z}} \mathbf{A}_{\alpha}, \alpha=r, 2,3$, or, equivalently, by

$$
\hat{\mathbf{Z}}=i \hat{\mathbf{L}}_{r 23} \hat{\mathbf{A}}_{r 23}^{-1}
$$

where $\hat{\mathbf{L}}_{r 23}$ and $\hat{\mathbf{A}}_{r 23}$ denote the $3 \times 3$ matrices whose columns are the components of the vectors $\mathbf{L}_{\alpha}$ and $\mathbf{A}_{\alpha}$, respectively, with $\alpha=r, 2,3$. As a result,

$$
\operatorname{det}(\hat{\mathbf{Z}}) \equiv-i \frac{\left\|\mathbf{L}_{r} \mathbf{L}_{2} \mathbf{L}_{3}\right\|}{\left\|\mathbf{A}_{r} \mathbf{A}_{2} \mathbf{A}_{3}\right\|}
$$

and the equivalence of equations (21) and (22) is evident.

The complex-valued solutions of the dispersion equation, for which either of $\omega$ and $k$ is complex, correspond to the leaky waves. In contrast, the passband SAWs are associated with real roots of the dispersion equation such that render both $\omega$ and $k$ real. Indeed, given real $\omega$ and $k$, the amplitude $b_{r}$ appearing in Eq. (20) vanishes according to the law of energy conservation or, formally, due to Eqs. (13), (15) and (20) which together yield $\left|b_{r}\right|^{2}=$ $\xi_{\text {leaky }}^{\dagger} \hat{\mathbf{T}} \xi_{\text {leaky }}=0$. Thus occurrence of a real root of the dispersion equation in the form (21) or (22) ensures the existence of two-partial passband SAW $\xi_{\text {saw }}=\sum_{\alpha=2,3} b_{\alpha} \xi_{\alpha}(y)$ which fulfills the traction-free condition

$$
b_{2} \mathbf{L}_{2}+b_{3} \mathbf{L}_{3}=\mathbf{0}
$$


on the surface of the phononic crystal. Note that vanishing of $\mathbf{L}_{2}$ or $\mathbf{L}_{3}$ may occur only at the expense of fulfilling certain additional conditions on top of Eq. (21) or Eq. (22).

The determinants in Eqs. (21) and (22) within the passbands are complexvalued functions, so we need to examine a possibility of simultaneous vanishing of the real and imaginary parts of $\left\|\mathbf{L}_{r} \mathbf{L}_{2} \mathbf{L}_{3}\right\|$ or of $\operatorname{det}(\hat{\mathbf{Z}})$ at some real $\omega$ and $k$. We shall analyze $\left\|\mathbf{L}_{r} \mathbf{L}_{2} \mathbf{L}_{3}\right\|$, since dividing $\left\|\mathbf{L}_{r} \mathbf{L}_{2} \mathbf{L}_{3}\right\|$ by $\left\|\mathbf{A}_{r} \mathbf{A}_{2} \mathbf{A}_{3}\right\|$ does not simplify the task.

Let us proceed from the equality

$$
\operatorname{Re}\left(\left\|\mathbf{L}_{r} \mathbf{L}_{2} \mathbf{L}_{3}\right\|\right)=0
$$

Given real $\omega$ and $k$, we can employ Eq. (18). Premultiplying (18) by the (non-zero) vector products $\mathbf{L}_{r} \times \mathbf{L}_{2}$ from the left and by $\mathbf{L}_{r}^{*} \times \mathbf{L}_{2}^{*}$ from the right results in the identity

$$
\left|\left\|\mathbf{L}_{i} \mathbf{L}_{r} \mathbf{L}_{2}\right\|\right|^{2}=-\left\|\mathbf{L}_{r} \mathbf{L}_{2} \mathbf{L}_{3}\right\| \cdot\left\|\mathbf{L}_{r}^{*} \mathbf{L}_{2}^{*} \mathbf{L}_{6}^{*}\right\|-\left\|\mathbf{L}_{r} \mathbf{L}_{2} \mathbf{L}_{6}\right\| \cdot\left\|\mathbf{L}_{r}^{*} \mathbf{L}_{2}^{*} \mathbf{L}_{3}^{*}\right\|
$$

In view of Eq. (26), this identity reduces to

$$
||\left|\mathbf{L}_{i} \mathbf{L}_{r} \mathbf{L}_{2} \|\right|^{2}=-2 \operatorname{Im}\left(\left\|\mathbf{L}_{r} \mathbf{L}_{2} \mathbf{L}_{3}\right\|\right) \operatorname{Im}\left(\left\|\mathbf{L}_{r} \mathbf{L}_{2} \mathbf{L}_{6}\right\|\right) .
$$

Therefore the relation

$$
\operatorname{Im}\left(\left\|\mathbf{L}_{r} \mathbf{L}_{2} \mathbf{L}_{3}\right\|\right)=0
$$

which we want to occur along with Eq. (26), cannot be fulfilled unless

$$
\left\|\mathbf{L}_{i} \mathbf{L}_{r} \mathbf{L}_{2}\right\|=0
$$

Equality (30) implies the vanishing of its generally independent real and imaginary parts. Thus this line of arguments suggests that the existence of 
a passband SAW should be conditioned by at least three real equations on the parameters of the problem: one is Eq. (26) and two others come from Eq. (30).

On the other hand, we may equally proceed from Eq. (29) and inspect the conditions for its simultaneous fulfillment along with (26) at some real $\omega$ and $k$. Then, acting similarly as above, we obtain the identity of the form

$$
\left|\left\|\mathbf{L}_{i} \mathbf{L}_{r} \mathbf{L}_{2}\right\|\right|^{2}=-2 R e\left(\left\|\mathbf{L}_{r} \mathbf{L}_{2} \mathbf{L}_{3}\right\|\right) \operatorname{Re}\left(\left\|\mathbf{L}_{r} \mathbf{L}_{2} \mathbf{L}_{6}\right\|\right) \text {. }
$$

This identity proposes that it is Eq. (26), rather than Eq. (29), which requires two conditions to be fulfilled. One could have thought on these grounds that Eqs. (26) and (29) are actually equivalent to two real equations each, thus providing four conditions in aggregate for a real root of (21) to occur. Another argument favoring the latter conjecture is that the collinearity of two complex vectors, as required for $\mathbf{L}_{2}$ and $\mathbf{L}_{3}$ by Eq. (25), is generally conditioned by four equalities. However, the vectors $\mathbf{L}_{2}$ and $\mathbf{L}_{3}$ are not arbitrary, since they are involved in the orthogonality and completeness relations. Altogether, it is seen that the above reasonings do not yet provide unequivocal conclusions and so we need to refine our analysis.

\subsection{Further analysis}

Denote by $X=\left(\omega, x_{j}\right)$ the real multidimensional space of frequency $\omega$ and some other parameters $x_{1}, x_{2}, \ldots$, such as the tangential wavenumber $k$, the orientation angles, the material constants of the layers and their thicknesses. Given $\omega$ and $k$ lying in a passband with two bulk modes, let us assume that some point $X_{0}=\left(\omega_{0}, x_{0 j}\right)$ of $X$ is a real root of Eq. (21) and hence the 
passband SAW exists at $X_{0}$. Once Eq. (21) is fulfilled at real $\omega$ and $k$, then so is Eq. (30), i.e. the vectors $\mathbf{L}_{i}, \mathbf{L}_{r}, \mathbf{L}_{2}$ are coplanar and so the equality

$$
d_{i} \mathbf{L}_{i}+d_{r} \mathbf{L}_{r}+d_{2} \mathbf{L}_{2}=\mathbf{0}
$$

holds true alongside (25). In other words, the passband SAW coexists with the wave field $\xi(y)=\sum_{\alpha=i, r, 2} d_{\alpha} \xi_{\alpha}(y)$ describing the reflection from the traction-free surface $y=0$ of the structure.

For the future use, we need to find explicitly the partial amplitudes $b_{\alpha}$ and $d_{\alpha}$. Premultiplying (18) from the right by the vector $\mathbf{L}_{r}^{*} \times \mathbf{L}_{2}^{*}$ first and by the vector $\mathbf{L}_{2}^{*} \times \mathbf{L}_{6}^{*}$ second, we obtain, respectively,

$$
b_{2}=\left\|\mathbf{L}_{r}^{*} \mathbf{L}_{2}^{*} \mathbf{L}_{5}^{*}\right\|, \quad b_{3}=\left\|\mathbf{L}_{r}^{*} \mathbf{L}_{2}^{*} \mathbf{L}_{6}^{*}\right\|
$$

and

$$
d_{i}=\left\|\mathbf{L}_{2}^{*} \mathbf{L}_{6}^{*} \mathbf{L}_{i}^{*}\right\|, \quad d_{r}=-\left\|\mathbf{L}_{2}^{*} \mathbf{L}_{6}^{*} \mathbf{L}_{r}^{*}\right\|, \quad d_{2}=\left\|\mathbf{L}_{2}^{*} \mathbf{L}_{6}^{*} \mathbf{L}_{5}^{*}\right\|,
$$

where we omit arbitrary constant factors. Note that setting $\mathbf{L}_{2} \neq \mathbf{0}$ ensures non-vanishing of the vector $\mathbf{L}_{2} \times \mathbf{L}_{6}$ and of the amplitudes $b_{3}, d_{i}, d_{r}$ at the point $X_{0}$ (to this end, see also the energy-based observations in the end of $\S 2)$.

Bearing in mind that Eq. (21) and hence Eq. (30) are considered valid at $X_{0}$, we are going to investigate their behavior in the vicinity of $X_{0}$. The left-hand sides of Eqs. (21) and Eq. (30) taken at $X=X_{0}+\Delta X$ become

$$
\left\|\mathbf{L}_{r} \mathbf{L}_{2} \mathbf{L}_{3}\right\|=\left(\left\|\mathbf{L}_{r} \frac{\partial \mathbf{L}_{2}}{\partial X_{0}} \mathbf{L}_{3}\right\|+\left\|\mathbf{L}_{r} \mathbf{L}_{2} \frac{\partial \mathbf{L}_{3}}{\partial X_{0}}\right\|\right) \Delta X+o(\Delta X)
$$

and

$$
\left\|\mathbf{L}_{i} \mathbf{L}_{r} \mathbf{L}_{2}\right\|=\left(\left\|\frac{\partial \mathbf{L}_{i}}{\partial X_{0}} \mathbf{L}_{r} \mathbf{L}_{3}\right\|+\left\|\mathbf{L}_{i} \frac{\partial \mathbf{L}_{r}}{\partial X_{0}} \mathbf{L}_{2}\right\|+\left\|\mathbf{L}_{i} \mathbf{L}_{r} \frac{\partial \mathbf{L}_{2}}{\partial X_{0}}\right\|\right) \Delta X+o(\Delta X)
$$


where $\mathbf{L}_{\alpha}=\mathbf{L}_{\alpha}\left(X_{0}\right)$ and $\partial \mathbf{L}_{\alpha} / \partial X_{0}$ are the derivatives $\partial / \partial X$ applied to $\mathbf{L}_{\alpha}=\mathbf{L}_{\alpha}(X)$ and taken at the point $X_{0}$. For brevity, we are not writing out these and following expressions in terms of components $\Delta X_{j}$ and $\partial / \partial X_{0 j}$.

The identity implied by either diagonal block of Eq. (16) allows us to represent each derivative $\partial \mathbf{L}_{\alpha} / \partial X_{0}$ as a linear combination of vectors $\mathbf{L}_{\alpha}=$ $\mathbf{L}_{\alpha}\left(X_{0}\right)$, namely,

$$
\begin{aligned}
\frac{\partial \mathbf{L}_{\alpha}}{\partial X_{0}}= & \left(\frac{\partial \mathbf{L}_{\alpha}^{t}}{\partial X_{0}} \mathbf{A}_{i}^{*}\right) \mathbf{L}_{i}-\left(\frac{\partial \mathbf{L}_{\alpha}^{t}}{\partial X_{0}} \mathbf{A}_{r}^{*}\right) \mathbf{L}_{r} \\
& +\sum_{\beta=2}^{3}\left[\left(\frac{\partial \mathbf{L}_{\alpha}^{t}}{\partial X_{0}} \mathbf{A}_{\beta}^{*}\right) \mathbf{L}_{\beta+3}+\left(\frac{\partial \mathbf{L}_{\alpha}^{t}}{\partial X_{0}} \mathbf{A}_{\beta+3}^{*}\right) \mathbf{L}_{\beta}\right]
\end{aligned}
$$

Substituting Eq. (37) in Eq. (35) and taking into account Eqs. (21), (32) and (33), we obtain

$$
\begin{aligned}
\left\|\mathbf{L}_{r} \mathbf{L}_{2} \mathbf{L}_{3}\right\|= & \left\{\sum _ { \beta = 2 } ^ { 3 } \left[\left(\frac{\partial \mathbf{L}_{2}^{t}}{\partial X_{0}} \mathbf{A}_{\beta}^{*}\right)\left\|\mathbf{L}_{r} \mathbf{L}_{\beta+3} \mathbf{L}_{3}\right\|\right.\right. \\
& \left.\left.+\left(\frac{\partial \mathbf{L}_{3}^{t}}{\partial X_{0}} \mathbf{A}_{\beta}^{*}\right)\left\|\mathbf{L}_{r} \mathbf{L}_{2} \mathbf{L}_{\beta+3}\right\|\right]\right\} \Delta X+o(\Delta X) \\
= & \left\{\sum_{\beta=2}^{3}\left[\left(\frac{\partial \mathbf{L}_{2}^{t}}{\partial X_{0}} \mathbf{A}_{\beta}^{*}\right) \frac{b_{\beta}^{*} b_{2}}{b_{3}}+\left(\frac{\partial \mathbf{L}_{3}^{t}}{\partial X_{0}} \mathbf{A}_{\beta}^{*}\right) b_{\beta}^{*}\right]\right\} \Delta X+o(\Delta X) \\
= & \frac{1}{b_{3}}\left(\mathbf{A}_{0}^{\dagger} \frac{\partial \mathbf{L}}{\partial X_{0}}\right) \Delta X+o(\Delta X)
\end{aligned}
$$

where $\mathbf{A}_{0}=\sum_{\alpha=2,3} b_{\alpha} \mathbf{A}_{\alpha}\left(X_{0}\right)$ and $\partial \mathbf{L} / \partial X_{0}$ implies the derivative $\partial / \partial X$ applied at the point $X_{0}$ to the vector $\mathbf{L}=\sum_{\alpha=2}^{3} b_{\alpha} \mathbf{L}_{\alpha}(X)$. The coefficients $b_{\alpha}$ in both sums above have constant values (33) referred to $X_{0}$. Similarly, inserting Eq. (37) in Eq. (36) and employing Eqs. (21), (32)-(34) yields

$$
\begin{aligned}
\left\|\mathbf{L}_{i} \mathbf{L}_{r} \mathbf{L}_{2}\right\| & =\frac{1}{d_{i}}\left(\mathbf{A}_{0}^{\dagger} \frac{\partial \overline{\mathbf{L}}}{\partial X_{0}}\right) \Delta X+o(\Delta X) \\
& =-\frac{1}{d_{i}}\left(\overline{\mathbf{A}}_{0}^{t} \frac{\partial \mathbf{L}^{*}}{\partial X_{0}}\right) \Delta X+o(\Delta X),
\end{aligned}
$$

where $\overline{\mathbf{A}}_{0}=\sum_{\alpha=i, r, 2} d_{\alpha} \mathbf{A}_{\alpha}\left(X_{0}\right)$ and $\partial \overline{\mathbf{L}} / \partial X_{0}$ is the derivative applied at $X_{0}$ to the vector $\overline{\mathbf{L}}=\sum_{\alpha=i, r, 2} d_{\alpha} \mathbf{L}_{\alpha}(X)$. The coefficients $d_{\alpha}$ in both sums above 
have constant values (34) referred to $X_{0}$. In the second equality in (39) we have used Eq. (15) and the fact that both $\mathbf{L}$ and $\overline{\mathbf{L}}$ vanish when they are referred to $X_{0}$.

In view of the remark below Eq. (33), $b_{3}$ defined at $X_{0}$ does not vanish, i.e. at least either its real or imaginary part is non-zero. Let $^{1} b_{3}^{\prime \prime} \equiv \operatorname{Im}\left(b_{3}\right) \neq 0$, which by $(33)_{2}$ implies that the function $\operatorname{Im}\left(\left\|\mathbf{L}_{r} \mathbf{L}_{2} \mathbf{L}_{6}\right\|\right)$ is non-zero at the point $X_{0}$ and hence also in some vicinity of $X_{0}$. Invoking Eq. (28), we can conclude that for $X$ close enough to $X_{0}$ the roots of the system of equations (26) and (30) are simultaneously the roots of Eq. (21). Recall that the occurrence of a real root of Eq. (21) is the criterion for existence of the passband SAW. Thus we need to analyze a possibility of common real roots of Eqs. (26) and (30) in the vicinity of $X_{0}$.

By virtue of $\mathbf{L}\left(X_{0}\right)=\mathbf{0}$ and Eqs. (14), (15), $R e\left(\mathbf{A}_{0}^{\dagger} \partial \mathbf{L} / \partial X_{0}\right)=0$. Hence, Eq. (38) implies that

$$
R e\left(\| \mathbf{L}_{r} \mathbf{L}_{2} \mathbf{L}_{3}||\right)=-i \frac{b_{3}^{\prime \prime}}{\left|b_{3}\right|^{2}}\left(\mathbf{A}_{0}^{\dagger} \frac{\partial \mathbf{L}}{\partial X_{0}}\right) \Delta X+o(\Delta X) .
$$

Using (39) and (40), the system of equations (26) and (30) can be represented near $X_{0}$ as follows:

$$
\left\{\begin{array}{l}
F(X) \equiv i\left(\mathbf{A}_{0}^{\dagger} \frac{\partial \mathbf{L}}{\partial X_{0}}\right) \Delta X+o(\Delta X)=0 \\
C(X) \equiv\left(\overline{\mathbf{A}}_{0}^{\dagger} \frac{\partial \mathbf{L}}{\partial X_{0}}\right) \Delta X+o(\Delta X)=0
\end{array}\right.
$$

where $F(X)$ is a real function and $C(X)$ is a complex-valued function, i.e. (41) is a system of three real equations in the variables $\Delta X=\left(\Delta \omega, \Delta x_{j}\right), j=$

\footnotetext{
${ }^{1}$ If $\operatorname{Im}\left(b_{3}\right)=0$, then the subsequent analysis is to be performed along the similar lines but proceeding from $\operatorname{Re}\left(b_{3}\right) \neq 0$ and using Eqs. (29), (31) instead of Eqs. (21), (28), respectively.
} 
$1,2, \ldots$ Unless a particular crystallographic symmetry of the constitutive layers is involved (see §4), these three equations are linearly independent, i.e. the coefficient matrix associated with system (41) has rank 3. Indeed, there is no apparent reason to expect that the $3 \times 3$ matrices

$$
\hat{\mathbf{W}}=\left(\begin{array}{ccc}
i\left(\mathbf{A}_{0}^{\dagger} \frac{\partial \mathbf{L}}{\partial \omega_{0}}\right) & i\left(\mathbf{A}_{0}^{\dagger} \frac{\partial \mathbf{L}}{\partial x_{01}}\right) & i\left(\mathbf{A}_{0}^{\dagger} \frac{\partial \mathbf{L}}{\partial x_{02}}\right) \\
\operatorname{Re}\left(\overline{\mathbf{A}}_{0}^{\dagger} \frac{\partial \mathbf{L}}{\partial \omega_{0}}\right) & \operatorname{Re}\left(\overline{\mathbf{A}}_{0}^{\dagger} \frac{\partial \mathbf{L}}{\partial x_{01}}\right) & \operatorname{Re}\left(\overline{\mathbf{A}}_{0}^{\dagger} \frac{\partial \mathbf{L}}{\partial x_{02}}\right) \\
\operatorname{Im}\left(\overline{\mathbf{A}}_{0}^{\dagger} \frac{\partial \mathbf{L}}{\partial \omega_{0}}\right) & \operatorname{Im}\left(\overline{\mathbf{A}}_{0}^{\dagger} \frac{\partial \mathbf{L}}{\partial x_{01}}\right) & \operatorname{Im}\left(\overline{\mathbf{A}}_{0}^{\dagger} \frac{\partial \mathbf{L}}{\partial x_{02}}\right)
\end{array}\right)
$$

would have a zero determinant for all possible choices of the parameters $x_{1}, x_{2}$ from the variety of problem parameters $x_{j}$. For example, one trivial possibility for vanishing of $\operatorname{det} \hat{\mathbf{W}}$ could be collinearity of $\mathbf{A}_{0}$ and $\overline{\mathbf{A}}_{0}$ (i.e. proportionality of $F(X)$ and $C(X)$ ), but such event is actually impossible, because invoking either of the diagonal blocks of (16) along with (25) and (32) shows that $\mathbf{A}_{0}=\mathbf{L}_{r}^{*} \times \mathbf{L}_{2}^{*}$ and $\overline{\mathbf{A}}_{0}=\mathbf{L}_{2}^{*} \times \mathbf{L}_{6}^{*}$, where the vectors $\mathbf{L}_{6}$ and $\mathbf{L}_{r}$ cannot be collinear and hence neither can $\mathbf{A}_{0}$ and $\overline{\mathbf{A}}_{0}$. Thus generally $\operatorname{det} \hat{\mathbf{W}} \neq 0$, in which case system (41) defines the branch of passband SAWs near the point $X_{0}$ as the increment $\left(\Delta \omega, \Delta x_{1}, \Delta x_{2}\right)$ of the frequency and an arbitrarily chosen pair of parameters under a slight variation of other parameters. An example of such generic situation where $\operatorname{det} \hat{\mathbf{W}} \neq 0$ is considered in $\S 6$, see the discussion around Fig. 7.

Some additional insight may be gained via invoking the time- and depthaveraged Lagrangian associated with the "auxiliary" wave field formed of decaying modes $\alpha=2,3$ with the amplitudes $b_{\alpha}$ (33) referred to $X_{0}$. The displacement-traction field of this wave on the surface $y=0$ is composed of the vectors $\mathbf{A}=\sum_{\alpha=2,3} b_{\alpha} \mathbf{A}_{\alpha}(X)$ and $\mathbf{L}=\sum_{\alpha=2,3} b_{\alpha} \mathbf{L}_{\alpha}(X)$. According to 
[26], the Lagrangian $£$ per unit surface can be developed as follows

$$
£(X)=-\left.\frac{1}{8} n_{i}\left(\sigma_{i j}^{*} u_{j}+\sigma_{i j} u_{j}^{*}\right)\right|_{y=0}=-\frac{i k}{8}\left(\mathbf{L}^{\dagger} \mathbf{A}-\mathbf{A}^{\dagger} \mathbf{L}\right)=\frac{i k}{4}\left(\mathbf{A}^{\dagger} \mathbf{L}\right),
$$

where the latter equality is due to Eq. (15). By (25) $\mathbf{L}=\mathbf{0}$ at the point $X_{0}$, hence

$$
\frac{\partial £}{\partial X_{0}}=\frac{i k}{4}\left(\mathbf{A}_{0}^{\dagger} \frac{\partial \mathbf{L}}{\partial X_{0}}\right)
$$

where $\partial £ / \partial X_{0}$ is the derivative $\partial / \partial X$ applied to $£(X)$ and taken at $X_{0}$. Inserting the expressions for partial derivatives resulting from (44) in (41), we arrive at the equivalent representation of the function $F(X)$ as

$$
F(X)=\frac{4}{k}\left(\frac{\partial £}{\partial \omega_{0}} \Delta \omega+\sum_{j} \frac{\partial £}{\partial x_{0 j}} \Delta x_{j}\right)+o(\Delta X) .
$$

Since $\omega_{0} \partial £ / \partial \omega_{0}=2 E_{k i n}$, where $E_{k i n}$ is the time- and depth-averaged kinetic energy, $\partial £ / \partial \omega_{S}$ is non-zero (positive) and so the equation $F(X)=0(41)_{1}$ with $F(X)$ given by (45) always allows one to express $\Delta \omega$ via $\Delta x_{j}$. Doing so and inserting the result in Eq. $(41)_{2}$ recasts the latter in the form

$$
\widetilde{C}\left(\Delta x_{j}\right) \equiv \sum_{j}\left(\overline{\mathbf{A}}_{0}^{\dagger} \widetilde{\mathbf{L}}_{j}\right) \Delta x_{j}+o(\Delta X)=0
$$

where

$$
\widetilde{\mathbf{L}}_{j}=\frac{\partial \mathbf{L}}{\partial x_{0 j}}-\frac{\partial £ / \partial x_{0 j}}{\partial £ / \partial \omega_{0}} \frac{\partial \mathbf{L}}{\partial \omega_{0}} .
$$

Equation (46) implies a system of two real equations in unknowns $\Delta x_{j}$. Consider the $2 \times 2$ coefficient matrices

$$
\hat{\mathbf{W}}^{\prime}=\left(\begin{array}{cc}
\operatorname{Re}\left(\overline{\mathbf{A}}_{0}^{\dagger} \widetilde{\mathbf{L}}_{1}\right) & \operatorname{Re}\left(\overline{\mathbf{A}}_{0}^{\dagger} \widetilde{\mathbf{L}}_{2}\right) \\
\operatorname{Im}\left(\overline{\mathbf{A}}_{0}^{\dagger} \widetilde{\mathbf{L}}_{1}\right) & \operatorname{Im}\left(\overline{\mathbf{A}}_{0}^{\dagger} \widetilde{\mathbf{L}}_{2}\right)
\end{array}\right)
$$


associated with different choices of $x_{1}, x_{2}$ from the variety of problem parameters $x_{j}$ involved in (46). Given the same choice of $x_{1}, x_{2}$ as in (42), the determinants of $\hat{\mathbf{W}}^{\prime}$ and of $\hat{\mathbf{W}}$ are related by the identity

$$
\operatorname{det} \hat{\mathbf{W}}=4 \frac{\partial £}{\partial \omega_{0}} \operatorname{det} \hat{\mathbf{W}}^{\prime}
$$

It is seen from (48) and (49) that vanishing of these determinants requires the two pairs of values $\operatorname{Re}\left(\overline{\mathbf{A}}_{0}^{\dagger} \widetilde{\mathbf{L}}_{j}\right)$ and $\operatorname{Im}\left(\overline{\mathbf{A}}_{0}^{\dagger} \widetilde{\mathbf{L}}_{j}\right)$ to be proportional to each other with the same factor for both $j=1,2$, which is an unlikely event, especially if it is to occur for all possible choices of $x_{1}, x_{2}$ from the variety of $x_{j}$. Hence three real equations in (41) or, equivalently, two real equations in (46) generally are linear independent.

Thus, having assumed the occurrence of passband SAW at some point $X_{0}=\left(\omega_{0}, x_{0 j}\right)$ of the multidimensional space of problem parameters $X=$ $\left(\omega, x_{j}\right)$, we showed that the existence of SAW in the vicinity of $X_{0}$ is conditioned by simultaneous fulfillment of the three real equations which normally are linearly independent. Since the point $X_{0}$ is selected arbitrarily from the existence space of the passband SAW, we can conclude that generally three conditions are to be fulfilled for a passband SAW to occur.

The previous discussion concerns non-leaky SAWs in the passbands with one pair of bulk modes. Let us consider possible occurrence of SAW in a passband where four partial modes are bulk ones. We will label the bulk incident and reflected modes by the indices $\alpha=i 1, i 2$ and $\alpha=r 1, r 2$, respectively, and the remaining two inhomogeneous modes by $\alpha=3,6$, specifying the decaying mode by $\alpha=3$. The non-leaky SAW in such passband may exist only as a single partial mode $\alpha=3$ and it must satisfy the equality 
$\mathbf{L}_{3}=0$. This is the case if the equation

$$
\left\|\mathbf{L}_{r 1} \mathbf{L}_{r 2} \mathbf{L}_{3}\right\|=0
$$

is fulfilled for real frequency and wavenumber. Indeed, at real $\omega$ and $k$ neither the vectors $\mathbf{L}_{r 1}$ and $\mathbf{L}_{r 2}$ can be collinear, nor the vectors $\mathbf{L}_{r 1}, \mathbf{L}_{r 2}$ and $\mathbf{L}_{3}$ can be coplanar. Let us assume that

$$
\operatorname{Re}\left(\left\|\mathbf{L}_{r 1} \mathbf{L}_{r 2} \mathbf{L}_{3}\right\|\right)=0
$$

for some real $\omega$ and $k$. Then using Eqs. (19) and (51) we obtain

$$
\left.||\left|\mathbf{L}_{i 1} \mathbf{L}_{r 1} \mathbf{L}_{r 2}\right|\right|^{2}+||\left|\mathbf{L}_{i 2} \mathbf{L}_{r 1} \mathbf{L}_{r 2} \|\right|^{2}=-2 \operatorname{Im}\left(\left\|\mathbf{L}_{r 1} \mathbf{L}_{r 2} \mathbf{L}_{3}\right\|\right) \operatorname{Im}\left(\left\|\mathbf{L}_{r 1} \mathbf{L}_{r 2} \mathbf{L}_{6}\right\|\right) .
$$

It is seen from (52) that vanishing of two complex-valued determinants, i.e., vanishing of four real functions, is required for $\operatorname{Im}\left(|| \mathbf{L}_{r 1} \mathbf{L}_{r 2} \mathbf{L}_{3} \|\right)=0$, where the latter condition must complement Eq. (51) to fulfil the dispersion equation (50). Thus at least five real equations must be simultaneously satisfied in order that SAW occurs in a passband with four bulk modes.

\section{Passband SAWs on phononic crystals with planes of crystallographic symmetry}

\subsection{Symmetry plane orthogonal to the boundary}

Assume that the sagittal plane $(\mathbf{m}, \mathbf{n})$ orthogonal to the boundary is a common symmetry plane for all layers, so that among the six partial modes of the type (3) the two modes have the displacement and traction vectors orthogonal to the plane $(\mathbf{m}, \mathbf{n})$ - the shear horizontal or SH modes, and the 
other four modes have the displacement and traction vectors parallel to the plane $(\mathbf{m}, \mathbf{n})$ - the sagittal modes. Note that both the wave equation and the traction-free boundary condition maintain mutual uncoupling of the $\mathrm{SH}$ and sagittal modes.

Because of the above-mentioned uncoupling, the one-partial SH SAWs occurring in the stopbands of a pair of $\mathrm{SH}$ modes should not be viewed as a passband SAW, even though the SH stopbands may freely overlap the passbands of sagittal modes. The SH SAWs in periodically layered or functionally graded substrates with various types of variation of material properties within a period were studied [9].

Let us examine possible occurrence of the sagittally polarized passband SAWs. For this purpose, consider a passband of the sagittal modes where we label the two bulk (incident and reflected) modes by $\alpha=i, r$ and the two inhomogeneous modes by $\alpha=2,5$, specifying the decaying mode by the subscript $\alpha=2$. The one-partial sagittal passband SAW must meet the traction-free boundary condition $\mathbf{L}_{2}=\mathbf{0}$. It holds true if the equation

$$
\left\|\mathbf{L}_{r} \mathbf{L}_{2}\right\|=0
$$

is fulfilled for real $\omega$ and $k$. Here the notation $\|\cdot\|$ implies the determinant of $2 \times 2$ matrix whose columns are the sagittal components of enclosed vectors. Assume that

$$
\operatorname{Re}\left(\left\|\mathbf{L}_{r} \mathbf{L}_{2}\right\|\right)=0
$$

is satisfied for some real $\omega$ and $k$. Premultiplying the "sagittal part" of Eq. (18) vectorially by $\mathbf{L}_{r}$ from the left and by $\mathbf{L}_{r}^{*}$ from the right and using Eq. 
(53), we arrive at the identity

$$
\left\|\left|\mathbf{L}_{r} \mathbf{L}_{2} \|\right|^{2}=-2 \operatorname{Im}\left(\left\|\mathbf{L}_{r} \mathbf{L}_{2}\right\|\right) \operatorname{Im}\left(\left\|\mathbf{L}_{r} \mathbf{L}_{5}\right\|\right) .\right.
$$

It is seen that vanishing of a complex-valued determinant is required for vanishing of $\operatorname{Im}\left(\left\|\mathbf{L}_{r} \mathbf{L}_{2}\right\|\right)$. Hence at least three real equations must be simultaneously satisfied for the occurrence of the sagittally polarized passband SAW.

\subsection{Symmetry plane parallel to the boundary}

Assume that the horizontal plane orthogonal to the vector $\mathbf{n}$, i.e. parallel to the boundary and the layer interfaces, is a common symmetry plane for all layers. This means that the material tensors of the layers are invariant with

respect to the transformation described by the $3 \times 3$ matrix $\hat{\boldsymbol{\Lambda}}=\hat{\mathbf{I}}-2 \mathbf{n} \times \mathbf{n}$. Using $\hat{\Lambda} \mathbf{n}=-\mathbf{n}, \hat{\mathbf{\Lambda}} \mathbf{m}=\mathbf{m}$ and taking into account that the Stroh matrix $\hat{\mathbf{N}}_{j}$ (5) of each layer satisfies the general relation (6), one finds [15]

$$
\hat{\mathbf{N}}_{j}=\hat{\mathbf{J}} \hat{\mathbf{N}}_{j}^{t} \hat{\mathbf{J}}, \hat{\mathbf{N}}_{j}=-\hat{\mathbf{K}} \hat{\mathbf{N}}_{j} \hat{\mathbf{K}}
$$

where

$$
\hat{\mathbf{J}}=\left(\begin{array}{cc}
\hat{\mathrm{O}} & -\hat{\boldsymbol{\Lambda}} \\
\hat{\mathbf{\Lambda}} & \hat{\mathrm{O}}
\end{array}\right), \quad \hat{\mathbf{K}}=\hat{\mathbf{T}} \hat{\mathbf{J}}=\left(\begin{array}{cc}
\hat{\Lambda} & \hat{\mathrm{O}} \\
\hat{\mathrm{O}} & -\hat{\Lambda}
\end{array}\right)
$$

so that $\hat{\mathbf{J}}^{t}=\hat{\mathbf{J}}^{-1}=-\hat{\mathbf{J}}$ and $\hat{\mathbf{K}}^{t}=\hat{\mathbf{K}}^{-1}=\hat{\mathbf{K}}$. Note that the three matrix identities (6), $(56)_{1}$ and $(56)_{2}$ for $\hat{\mathbf{N}}_{j}$ are interrelated in the sense that joint consideration of any two of them yields the third one as a corollary.

It follows from (56) that the transfer matrix $\hat{\mathbf{M}}$ (8) obeys, besides the general property (11), also the identities [15]

$$
\hat{\mathbf{M}}^{-1}=\hat{-\mathbf{J}} \hat{\mathbf{M}}^{t} \hat{\mathbf{J}}, \hat{\mathbf{M}}=\hat{\mathbf{K}} \hat{\mathbf{M}}^{*} \hat{\mathbf{K}}
$$


where $(58)_{2}$, like [15], assumes real $\hat{\mathbf{N}}_{j}$ and hence real $\omega, k$. Accordingly to the above remark, we note that a conjunction of any two of the interrelated equalities (11), (58) ${ }_{1}$ and $(58)_{2}$ for $\hat{\mathbf{M}}$ can be taken as a sufficient prerequisite for analyzing the additional properties of the eigenvalues and eigenvectors of $\hat{\mathbf{M}}$ arising due to the presence of a horizontal symmetry plane.

Appealing to Eq. $(58)_{2}$ shows that $\hat{\mathbf{M}} \zeta_{\alpha}=\gamma_{\alpha} \zeta_{\alpha}$ leads to $\hat{\mathbf{M}} \hat{\mathbf{K}} \zeta_{\alpha}^{*}=\gamma_{\alpha}^{*} \hat{\mathbf{K}} \zeta_{\alpha}^{*}$, i.e., if $\gamma_{\alpha}$ and $\zeta_{\alpha}$ are the eigenvalue and eigenvector of $\hat{\mathbf{M}}$ then so are $\gamma_{\alpha}^{*}$ and $\hat{\mathbf{K}} \zeta_{\alpha}^{*}$. Hence the vector $\zeta_{\alpha}$ must be collinear with the vector $\hat{\mathbf{K}} \zeta_{\beta}^{*}$ such that corresponds to the eigenvalue $\gamma_{\beta}=\gamma_{\alpha}^{*}$. This link must hold along with the orthogonalities (13) and (15) following from (11). In consequence, $\zeta_{\alpha}^{t} \hat{\mathbf{J}} \zeta_{\beta}=0$ if and only if $\gamma_{\alpha} \neq \gamma_{\beta}^{*}$.

It is suitable to detail the above-mentioned result for the cases of bulk and inhomogeneous modes separately. For the bulk modes it follows that the general relations (12) and (13) are to be complemented by the relations

$$
\gamma_{i}=\gamma_{r}^{*}
$$

and

$$
\zeta_{i}=\hat{\mathbf{K}} \zeta_{r}^{*}
$$

Note that specifying proportionality as an equality in (60) leads to the equivalence of the normalizations of both $\zeta_{i}$ and $\zeta_{r}$ imposed in (13) to the condition $\zeta_{i}^{t} \hat{\mathbf{J}} \zeta_{r}=1$

Regarding the inhomogeneous modes, there are two possible cases which correspond to the so-called direct and indirect gaps. The first case is where all four $\gamma_{\alpha}$ are real, so that Eqs. (14) and (15) are to be complemented by the relations

$$
\gamma_{\alpha}=\gamma_{\alpha}^{*}, \quad \alpha=2,3
$$


and

$$
\zeta_{\alpha}=\hat{\mathbf{K}} \zeta_{\alpha}^{*}, \quad \zeta_{\alpha+3}=-\hat{\mathbf{K}} \zeta_{\alpha+3}^{*}, \quad \alpha=2,3
$$

where setting proportionality as an equality makes the normalization imposed in (15) equivalent to the condition $\zeta_{\alpha+3}^{t} \hat{\mathbf{J}} \zeta_{\alpha}=1$.

The second possible case is where the eigenvalues $\gamma_{\alpha}$ satisfying Eq. (14) are arranged in such a way that

$$
\gamma_{2}=\gamma_{3}^{*}=1 / \gamma_{5}^{*}=1 / \gamma_{6}
$$

and so Eq. (15) is to be complemented by the relations

$$
\zeta_{2}=\hat{\mathbf{K}} \zeta_{3}^{*}, \quad \zeta_{5}=-\hat{\mathbf{K}} \zeta_{6}^{*}
$$

in which setting proportionality as an equality makes the normalization imposed in (15) equivalent to the conditions $\xi_{6}^{t} \hat{\mathbf{J}} \zeta_{2}=1$ and $\xi_{5}^{t} \hat{\mathbf{J}} \zeta_{3}=1$.

Let us now return to our main issue and analyze possible existence of passband SAWs propagating along an arbitrary direction $\mathbf{m}$ on the boundary surface parallel to the symmetry plane. Consider a passband with two bulk modes and recall the dispersion equation for a passband SAW in the equivalent forms (21) and (41). Addressing the case (61), it is seen that Eqs. (60) and (62) yield

$$
\mathbf{L}_{i}=-\hat{\Lambda} \mathbf{L}_{r}^{*}, \quad \mathbf{L}_{\alpha}=-\hat{\Lambda} \mathbf{L}_{\alpha}^{*}, \quad \alpha=2,3
$$

Hence the determinant $\left\|\mathbf{L}_{i} \mathbf{L}_{r} \mathbf{L}_{2}\right\|$ proportional to $C(X)$ (see Eq. (39)) is a pure imaginary function and so (41) reduces to a system of two real equations. In the case (62), we observe from Eq. (64) that

$$
\mathbf{L}_{2}=-\hat{\Lambda} \mathbf{L}_{3}^{*}, \quad \mathbf{L}_{5}=-\hat{\Lambda} \mathbf{L}_{5}^{*}
$$


and then we use these relations along with $\mathbf{L}_{i}=-\hat{\mathbf{\Lambda}} \mathbf{L}_{r}^{*}$ to obtain

$$
\left\|\mathbf{L}_{r}^{*} \mathbf{L}_{2}^{*} \mathbf{L}_{3}^{*}\right\|=-\left\|\mathbf{L}_{i} \mathbf{L}_{2} \mathbf{L}_{3}\right\|, \quad\left\|\mathbf{L}_{i}^{*} \mathbf{L}_{r}^{*} \mathbf{L}_{2}^{*}\right\|=-\left\|\mathbf{L}_{i} \mathbf{L}_{r} \mathbf{L}_{3}\right\|
$$

Employing the latter equalities and the identity $2 \mathbf{L}_{\alpha} \times \mathbf{L}_{\beta}=\left(\mathbf{L}_{\alpha}-\mathbf{L}_{\beta}\right) \times$ $\left(\mathbf{L}_{\alpha}+\mathbf{L}_{\beta}\right)$, one can show that fulfillment of the dispersion equation (21) is subject to two real conditions specified, e.g., in the form

$$
\operatorname{Re}\left(\left\|\mathbf{L}_{r} \mathbf{L}_{2} \mathbf{L}_{3}\right\|=0, \quad \operatorname{Re}\left(\left\|\mathbf{L}_{i} \mathbf{L}_{r} \mathbf{L}_{2}\right\|\right)=0\right.
$$

Thus both possible cases considered above lead to the same conclusion that the existence of passband SAWs on a phononic crystal with a symmetry plane parallel to the boundary is conditioned by two real equations - rather than three ones as it is generally required in the absence of crystallographic symmetry. Note that, in agreement with the considerations of $\S 3.2$, it can be shown that the determinant of the matrix $\hat{\mathbf{W}}$ (42) turns to zero, provided that the parameters $x_{1}$ and $x_{2}$ are chosen so that their variation obeys the given symmetry.

Let us now consider a possibility for SAW to occur in a passband with four bulk modes specifically in the presence of a horizontal symmetry plane. In this case, the eigenvalues $\gamma_{3}=1 / \gamma_{6}^{*}$ associated with the available pair of inhomogeneous modes are necessarily real, i.e. $\gamma_{3}=\gamma_{3}^{*}=1 / \gamma_{6}$, and hence, by analogy with Eq. (62), we can take

$$
\zeta_{3}=\hat{\mathbf{K}} \zeta_{3}^{*}, \quad \zeta_{6}=-\hat{\mathbf{K}} \zeta_{6}^{*}
$$

Let the index $\alpha=3$ correspond to the decaying mode, so the boundary condition for the SAW in question is $\mathbf{L}_{3}=\mathbf{0}$. It is seen from Eq. (69) that $\mathbf{L}_{3}=-\hat{\Lambda} \mathbf{L}_{3}^{*}$, hence the normal component of $\mathbf{L}_{3}$ is real and the other 
two components are pure imaginary. Therefore vanishing of $\mathbf{L}_{3}$ and hence the existence of SAW is conditioned by three real equations only, instead of five equations which are required for the similar SAW in the case of general anisotropy.

The same conclusion can be drawn by invoking the bulk modes. The eigenvalues $\gamma_{\alpha}$ associated with the bulk modes $\alpha=i 1, i 2, r 1, r 2$ occur in complex conjugate pairs and the corresponding indices can be arranged as follows:

$$
\gamma_{i 1}=\gamma_{r 1}^{*}, \quad \gamma_{i 2}=\gamma_{r 2}^{*}
$$

The option $\gamma_{i 1}=\gamma_{i 2}^{*}, \gamma_{r 1}=\gamma_{r 2}^{*}$ is ruled out because it would lead to $\zeta_{i 1}=$ $\hat{\mathbf{K}} \zeta_{i 2}^{*}, \zeta_{r 1}=\hat{\mathbf{K}} \zeta_{r 2}^{*}$ and hence to $\zeta_{i 1}^{\dagger} \hat{\mathbf{T}} \zeta_{i 1}=-\zeta_{i 2}^{\dagger} \hat{\mathbf{T}} \zeta_{i 2}, \zeta_{r 1}^{\dagger} \hat{\mathbf{T}} \zeta_{r 1}=-\zeta_{r 2}^{\dagger} \hat{\mathbf{T}} \zeta_{r 2}$, which is a contradiction since such quadratic forms associated with the normal energy flux cannot be of different signs for both incident or both reflected modes. Similarly to Eqs. (59) and (60), it follows from (70) that we can take

$$
\zeta_{i 1}=\hat{\mathbf{K}} \zeta_{r 1}^{*}, \quad \zeta_{i 2}=\hat{\mathbf{K}} \zeta_{r 2}^{*}
$$

hence $\mathbf{L}_{i 1}=-\hat{\Lambda} \mathbf{L}_{r 1}^{*}$ and $\mathbf{L}_{i 2}=-\hat{\mathbf{\Lambda}} \mathbf{L}_{r 2}^{*}$. Taking into account these relations and also the above-obtained $\mathbf{L}_{3}=-\hat{\Lambda} \mathbf{L}_{3}^{*}$ yields

$$
\begin{aligned}
& \left\|\mathbf{L}_{i 1}^{*} \mathbf{L}_{r 1}^{*} \mathbf{L}_{r 2}^{*}\right\|=\left\|\mathbf{L}_{r 1} \mathbf{L}_{r 1} \mathbf{L}_{i 2}\right\|,\left\|\mathbf{L}_{i 2}^{*} \mathbf{L}_{r 1}^{*} \mathbf{L}_{r 2}^{*}\right\|=\left\|\mathbf{L}_{r 2} \mathbf{L}_{i 1} \mathbf{L}_{r 2}\right\| \\
& \left\|\mathbf{L}_{r 1}^{*} \mathbf{L}_{r 2}^{*} \mathbf{L}_{3}^{*}\right\|=-\left\|\mathbf{L}_{i 1} \mathbf{L}_{i 2} \mathbf{L}_{3}\right\| .
\end{aligned}
$$

Using identities (72) $)_{1}$ and $(72)_{2}$ allows one to express $\mathbf{L}_{i 1}$ and $\mathbf{L}_{i 2}$ via $\mathbf{L}_{r 1}$ and $\mathbf{L}_{r 2}$ by means of two real equations out of four ones listed in (52). On inserting these expressions and identity (72) 3 into $\left\|\mathbf{L}_{r 1} \mathbf{L}_{r 2} \mathbf{L}_{3}\right\|$, the latter determinant becomes proportional to its real part. Thus the equation $\left\|\mathbf{L}_{r 1} \mathbf{L}_{r 2} \mathbf{L}_{3}\right\|=0$, which is the dispersion equation (50) for one-partial passband SAW, can 
be fulfilled by satisfying two equations from (52) along with the equation $\operatorname{Re}\left(\left\|\mathbf{L}_{r 1} \mathbf{L}_{r 2} \mathbf{L}_{3}\right\|\right)=0$, i.e. three real equations in total, as we intended to show.

We complete this section by two brief remarks concerning other symmetry settings. The first is the case where all layers of the structure possess a common symmetry plane orthogonal to the direction of propagation $\mathbf{m}$. All the conclusions obtained above in $\S 4.2$ can be extended to this case merely by utilizing the matrix $\hat{\boldsymbol{\Lambda}}=\hat{\mathbf{I}}-2 \mathbf{m} \times \mathbf{m}$ in place of $\hat{\boldsymbol{\Lambda}}=\hat{\mathbf{I}}-2 \mathbf{n} \times \mathbf{n}$. The second case is where the symmetry plane parallel to the layer interfaces or perpendicular to the direction of propagation coexists with another plane of symmetry which is the sagittal plane. In this case, by (69), one of the two components of the sagittally polarized tractions is real and the other is pure imaginary. Hence the one-partial sagittal SAWs must satisfy only two real equations. In particular, this conclusion applies to the phononic crystals composed of elastically isotropic layers.

\section{$5 \quad$ Passband SAWs on phononic crystals with a symmetric period}

Assume that the period of a phononic crystal consists of an odd number $n=2 m+1$ of arbitrarily anisotropic layers, and that the layers $i$ and $n+1-i$

$(i=1, \ldots, m)$ are pairwise identical. We will call such period symmetric, in the sense that its arrangement is invariant to reflection about the midplane of the central layer. An example is a bilayered half-space, in which the thickness of the external layer is half the thickness of the internal layers of the same 
material and hence the period consisting of three layers is symmetric.

Denote the transfer matrix through a symmetric period by $\hat{\mathbf{M}}_{S}$ (with the label $S$ for symmetric). Due to the aforementioned ordering of layers in a symmetric period, the definition (8) specializes for the matrix $\hat{\mathbf{M}}_{S}$ as

$$
\begin{aligned}
\hat{\mathbf{M}}_{S} & =\hat{\mathbf{M}}_{1} \hat{\mathbf{M}}_{2} \cdots \hat{\mathbf{M}}_{m+1} \cdots \hat{\mathbf{M}}_{2} \hat{\mathbf{M}}_{1} \\
& =\mathrm{e}^{i k h_{1} \hat{\mathbf{N}}_{1}} \mathrm{e}^{i k h_{2} \hat{\mathbf{N}}_{2}} \cdots \mathrm{e}^{i k h_{m+1} \hat{\mathbf{N}}_{m+1}} \cdots \mathrm{e}^{i k h_{2} \hat{\mathbf{N}}_{2}} \mathrm{e}^{i k h_{1} \hat{\mathbf{N}}_{1}}
\end{aligned}
$$

Given real $\hat{\mathbf{N}}_{j}$ (hence real $\omega$ and $k$ ), using Eq. (73) and then complementing it by the general property (11) provides, respectively, the relations $[26,37]$

$$
\hat{\mathbf{M}}_{S}^{-1}=\hat{\mathbf{M}}_{S}^{*}, \quad \hat{\mathbf{M}}_{S}=\hat{\mathbf{T}} \hat{\mathbf{M}}_{S}^{t} \hat{\mathbf{T}}
$$

Similarly to the case of a horizontal symmetry plane, we note that any two of Eqs. (11), (74) 1 and $(74)_{2}$ considered jointly yield the third one as a corollary, and hence taking any pair of these equalities suffices for analyzing the properties of the eigenvalues and eigenvectors of $\hat{\mathbf{M}}_{S}$. For instance, consider first Eq. (74) $)_{1}$. It shows that if $\gamma_{\alpha}$ and $\zeta_{\alpha}$ are the eigenvalue and eigenvector of $\hat{\mathbf{M}}_{S}$ then so are $1 / \gamma_{\alpha}^{*}$ and $\zeta_{\alpha}^{*}$, hence the vector $\zeta_{\alpha}$ must be collinear with the vector $\zeta_{\beta}^{*}$ such that corresponds to the eigenvalue $\gamma_{\beta}=1 / \gamma_{\alpha}^{*}$. Let us specify this link for the bulk incident and reflected modes and for the inhomogeneous modes in the form of equalities

$$
\zeta_{i}=\zeta_{i}^{*}, \zeta_{r}=-\zeta_{r}^{*}
$$

and

$$
\zeta_{\alpha}=\zeta_{\alpha+3}^{*}
$$

respectively. Inserting them in Eqs. (13) and (15) following from (11) provides the orthonormalization condition

$$
\zeta_{\alpha}^{t} \hat{\mathbf{T}} \zeta_{\beta}=\delta_{\alpha, \beta}, \quad \alpha, \beta=1, \ldots, 6
$$


and the corresponding completeness relation

$$
\sum_{\alpha=1}^{3}\left(\zeta_{\alpha} \otimes \hat{\mathbf{T}} \zeta_{\alpha}+\zeta_{\alpha+3} \otimes \hat{\mathbf{T}} \zeta_{\alpha+3}\right)=\left(\begin{array}{cc}
\hat{\mathbf{I}} & \hat{\mathbf{O}} \\
\hat{\mathbf{O}} & \hat{\mathbf{I}}
\end{array}\right)
$$

Thus the assumed symmetric arrangement of layers does not affect the eigenvalues of the transfer matrix, but sets additional relations between its eigenvectors.

Note that the described algebraic properties of the transfer matrix $\hat{\mathbf{M}}_{S}$ through a symmetric period of a phononic crystal are the same as the corresponding properties of the transfer matrix for a homogeneous material, since the both transfer matrices, being of course explicitly different, satisfy the same relations of the form (11) and (74). As a result, the main features of the passband SAWs in the case of a symmetric period are quite similar to those of the supersonic SAWs in a homogeneous anisotropic substrate, and therefore we will only briefly outline them.

Consider a passband, in which there are two bulk modes $\alpha=i, r$ and four inhomogeneous modes $\alpha=2,3,5,6$. Let the indices $\alpha=2,3$ correspond to the decaying modes, so that the traction-free boundary condition for the twopartial passband SAW demands collinearity of the traction vectors $\mathbf{L}_{2}$ and $\mathbf{L}_{3}$. By using Eqs. (76), (77) and the left off-diagonal block of Eq. (78) the same way as it was done for the case of a homogeneous medium [30], it can be shown that if the vectors $\mathbf{L}_{2}$ and $\mathbf{L}_{3}$ are collinear, then so are the vectors $\mathbf{L}_{i}$ and $\mathbf{L}_{r}$ and, moreover, that these two pairs must satisfy the relations

$$
\mathbf{L}_{2} \pm i \mathbf{L}_{3}=0, \quad \mathbf{L}_{i} \pm i \mathbf{L}_{r}=0
$$

where the signs are not correlated. It is seen that Eqs. (79) differ from the similar Eqs. (25) and (32)-(34) which were obtained for the general 
case of an arbitrary (asymmetric) period. At the same time, the symmetry of period arrangement does not affect the general conclusion that in the absence of crystallographic symmetry the existence of non-leaky SAWs in the passbands with four bulk modes is conditioned by three real equations. This can be confirmed by way of manipulating Eqs. (75)-(78) similarly as in the case of supersonic SAWs in a homogeneous medium, see [33]. Symmetry of a period also does not affect another conclusion of the present paper, which is that the number of equations conditioning the existence of passband SAWs reduces to two in the presence of a common symmetry plane parallel to the boundary or orthogonal to the direction of propagation.

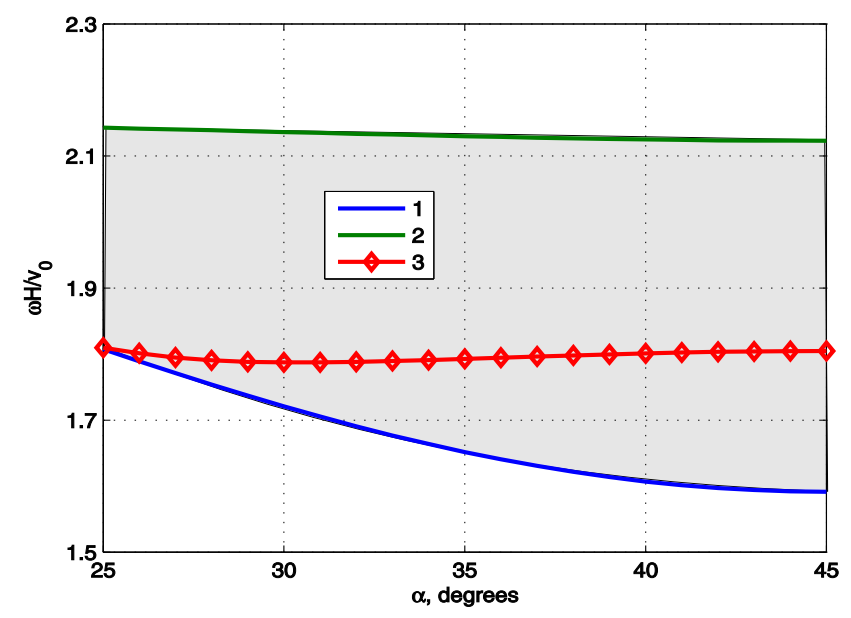

Figure 1: ranch of leaky wave in GaAs/AlAs at $k H=2$ on surface (001). Grey zone - passband. Curve 1 - lower edge of the passband, curve 2 - upper edge of the passband, curve 3 - real part of the parameter $\omega H / v_{0}$ of leaky waves.

Finally, we note that if the period is symmetric, then no SAWs can exist 
in the passbands with four bulk modes. Indeed, in this case a passband SAW must be composed by a single partial mode - say, $\alpha=3$ - and thus must satisfy the equality $\mathbf{L}_{3}=0$, but this would mean that $\zeta_{3}^{t} \hat{\mathbf{T}} \zeta_{3}=0$ which is impossible in view of Eq. (77) and of the fact that the eigenvalue $\gamma_{3}$ is always a distinct one inside a passband with four bulk modes.

\section{$6 \quad$ Numerical examples}

According to $§ 3.1$, the dispersion equation for passband SAWs may be cast in two equivalent forms (21) and (22). The former was used as the starting point for our analysis of the existence of passband SAWs, while the latter, $\operatorname{det}(\hat{\mathbf{Z}})=0$ where $\hat{\mathbf{Z}}$ is the surface impedance matrix defined in (23), appears suitable for practical computations. We have shown in $\S 3$ that the existence of two-partial passband SAWs on a phononic crystal of arbitrary anisotropy is generally conditioned by three real equations. In view of this result, provided that no crystallographic symmetry is involved, we consider the matrix $\hat{\mathbf{Z}}$ as a complex function of complex frequency $\omega=\omega^{\prime}-i \omega^{\prime \prime}$ and two real parameters $x_{1}, x_{2}$, given the values of the other parameters $x_{j}, j=3,4, \ldots$. The frequency is computed from the equation $\operatorname{det}(\hat{\mathbf{Z}})=0$ and simultaneously $\omega^{\prime \prime}$ is minimized by varying $x_{1}, x_{2}$. The minimum value of $\omega^{\prime \prime}$ is to be close to the computer zero. In this way, we find the values of real frequency and of $x_{1}, x_{2}$, at which the passband SAW exists for the given set of $x_{j}, j=3,4, \ldots$ If the structure possesses a symmetry plane orthogonal to the vectors $\mathbf{n}$ or $\mathbf{m}$ and so the existence of two-partial passband SAWs is conditioned by two

equations, then we consider $\hat{\mathbf{Z}}$ as a function of frequency and one of the pa- 
rameters, provided that its variation and the specified values of the other parameters do not break the given symmetry of the wave propagation geometry, i.e. either $\mathbf{n}$ or $\mathbf{m}$ remains orthogonal to the corresponding symmetry plane.

The numerical examples will be computed below for GaAs/AlAs and InAs/GaSb half-infinite periodically bilayered structures, each with equal thicknesses of the layers. The corresponding material constants are taken from site [38]. Unless otherwise specified, the layer interfaces and the boundary surface are parallel to the plane (001) which is a symmetry plane for both structures. In the following figures, the orientation of the propagation direction $\mathbf{m}$ along the given plane (001) is defined by the angle $\alpha$, which is counted off from direction [100]. As the frequency parameter, we will use the dimensionless quantity $\omega H / v_{0}$ where $H$ stands for the period and the value $v_{0}$ is equal to $3.33 \times 10^{3} \mathrm{~m} / \mathrm{s}$.

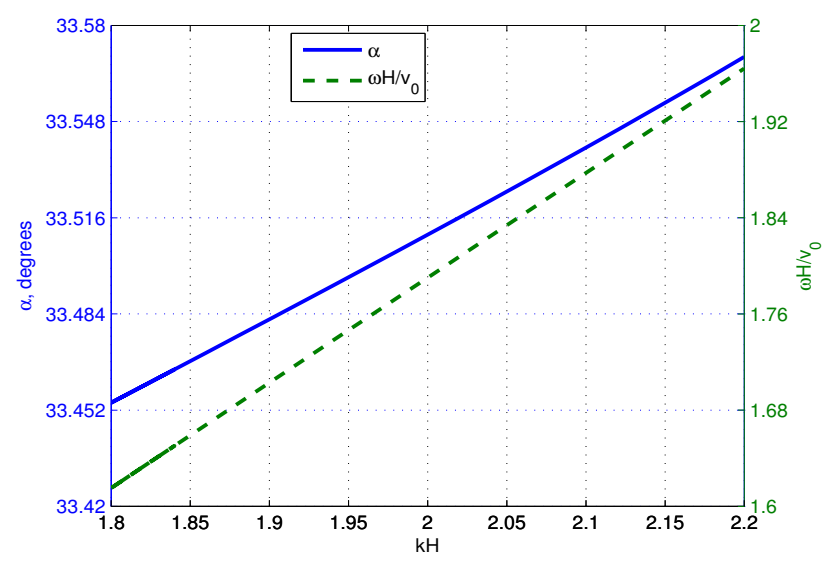

Figure 2: Branch of passband SAWs in GaAs/AlAs on surface (001). 
As it was mentioned in $\S 1$, Ref. [17] has reported on passband SAWs on the GaAs/AlAs superlattice. In particular, when the exterior layer is GaAs and the value of the parameter $k H$ equals 2 , there is a direction on the plane (001), along which the leaky wave, having the real part of its frequency in the lowest passband, propagates with zero attenuation and thus transforms into a passband SAW [17]. Our computations confirm this result. Figure 1 shows the branch of leaky waves in the passband of GaAs/AlAs as the dependence of the real part of frequency $\omega$ on the propagation angle $\alpha$ at fixed $k H=2$. The leaky wave becomes the passband SAW at $\alpha \approx 33.5^{\circ}$, in agreement with Ref. [17].

Since the boundary (001) is a symmetry plane and hence the corresponding dispersion equation for passband SAWs is equivalent to a system of two real equations, we can determine both the frequency $\omega$ and the propagation angle $\alpha$ of passband SAWs as (interrelated) functions of $k H$. The corresponding curves calculated within the interval $1.8 \leq k H \leq 2.2$ are plotted in Fig. 2. Note in passing that $\alpha$ hardly changes with varying $k H$.

Next we consider the superlattices InAs/GaSb and GaSb/InAs, where it is implied that the exterior layer is InAs in the former case and it is GaSb in the latter case. Figures 3 and 4 present the branches of leaky waves in the passband of InAs/GaSb and GaSb/InAs as the dependencies of the real part of $\omega$ on the angle $\alpha$ at $k H=1.8$ and at $k H=2.2$. Our computation reveals the existence of passband SAWs in both InAs/GaSb and $\mathrm{GaSb} / \mathrm{InAs}$ structures. The mutually related branches $\omega(k H)$ and $\alpha(k H)$ of these passband SAWs are shown in Figs. 5 and 6 .

Note that the angle $\alpha=45^{\circ}$ defines the orientation of the propagation 


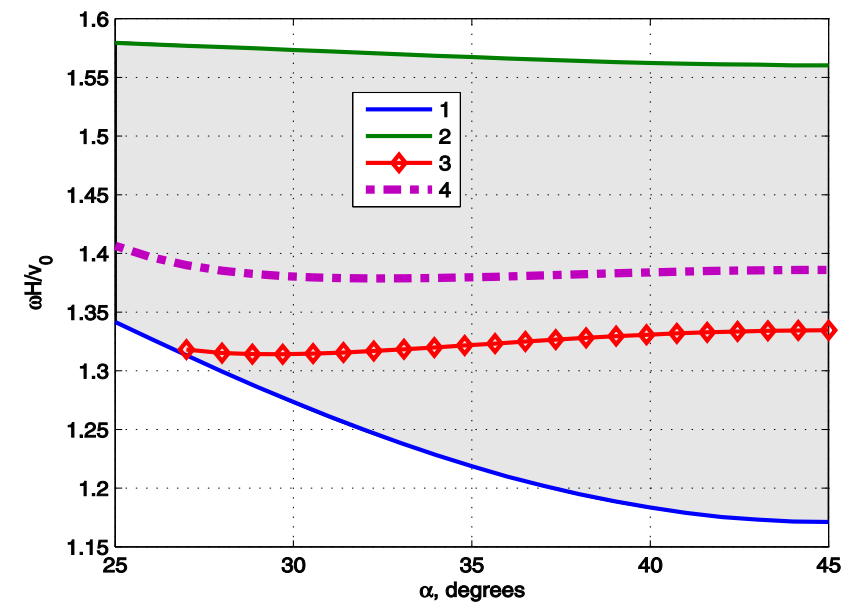

Figure 3: Branches of leaky waves in InAs/GaSb and GaSb/InAs on surface (001) at $k H=1.8$. Grey zone - passband. Curve 1 - lower edge of the passband, curve 2 - upper edge of the passband, curve 3 - real part of the parameter $\omega H / v_{0}$ of leaky waves in InAs/GaSb, 4 - real part of the parameter $\omega H / v_{0}$ of leaky waves in GaSb/InAs.

direction $\mathbf{m}$, for which the sagittal plane $(\mathbf{m}, \mathbf{n})$ becomes parallel to the symmetry plane of the superlattices under study. Given this orientation, each of the leaky waves appearing in Figs. 1, 3 and 4 transforms into a twopartial SAW with purely sagittal polarization which is fully uncoupled from the SH modes (see §4.1). Since this SAW is two-partial, its frequency and wavenumber lie in the stopband of sagittally polarized modes and hence, though this stopband actually overlaps the passband of SH modes, such sagittal SAW should be attributed to SAWs occurring in a full stopband.

The dependencies of $\omega$ and of $\alpha$ on $k H$ presented within the interval $1.8 \leq k H \leq 2.2$ in Figs. 2, 5 and 6 are almost linear. Therefore these 


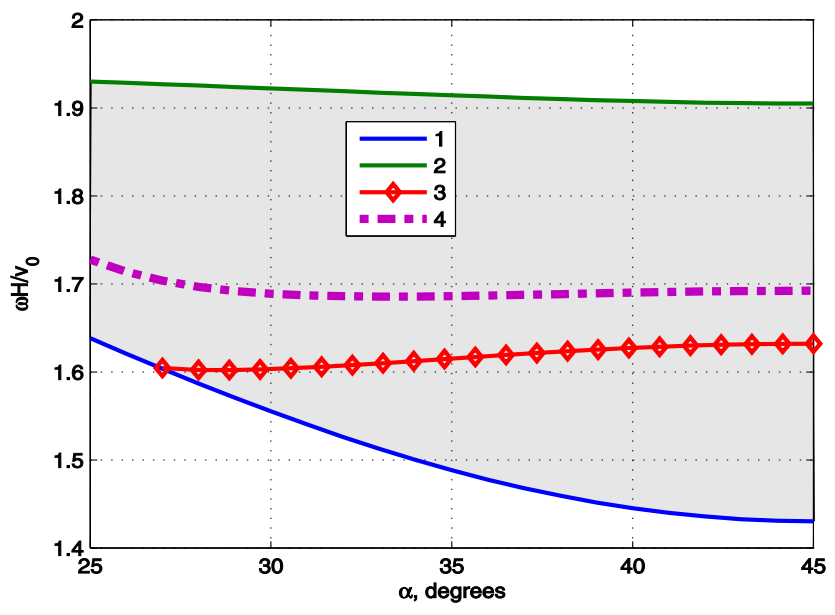

Figure 4: Branches of leaky waves in InAs/GaSb and GaSb/InAs on surface (001) at $k H=2.2$. Grey zone - passband. Curve 1 - lower edge of the passband, curve 2 - upper edge of the passband, curve 3 - real part of the parameter $\omega H / v_{0}$ of leaky waves in InAs/GaSb, 4 - real part of the parameter $\omega H / v_{0}$ of leaky waves in GaSb/InAs.

dependences can be calculated with a good accuracy from the approximate linear equations implied by Eq. (41) (where one can use (41) 1 and one of $(41)_{2}$ since the surface (001) is the symmetry plane). Applying this approach, for instance, in the case of GaSb/InAs has demonstrated that the approximate values of the frequency and of the propagation angle obtained from the linear part of Eq. (41) differ from their exact values only in the fifth digit and third digit, respectively.

Let us now look at the GaSb/InAs superlattice composed of the layers with a generic orientation of their interfaces which is not parallel to the symmetry plane. This new orientation is reached by performing three conse- 


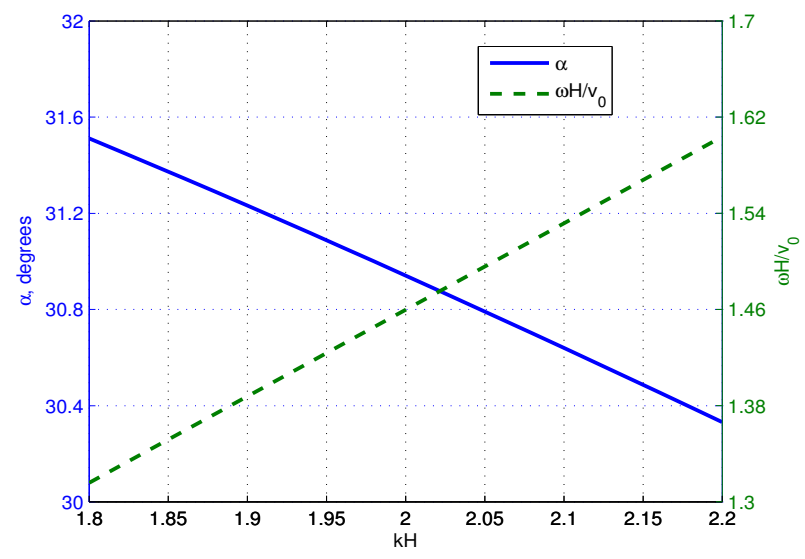

Figure 5: Branch of passband SAWs in InAs/GaSb on surface (001).

quential rotations starting from the initial symmetric orientation where the normal to the boundary $\mathbf{n}$ (y-axis) is [001] and the direction of propagation $\mathbf{m}$ ( $x$-axis) is [100]: the first rotation is about $y$-axis by angle $\alpha$, the second rotation is about new $x$-axis by angle $\beta$, and the third rotation is about new $z$-axis by angle $\gamma$. In terms of these three angles, the initial orientation can be referred to as $(\alpha, 0,0)$. Let us fix $k H=2$ and seek the passband SAWs for the frequency $\omega$ close to $\omega_{0}$ and the orientations close to $\left(\alpha_{0}, 0,0\right)$, where $\omega_{0}$ and $\alpha_{0}$ are the values for which the SAW with $k H=2$ exists on the GaSb/InAs of the initial symmetric orientation, see Fig. 6. Direct calculation shows that the determinant of matrix $\hat{\mathbf{W}}(42)$ does not vanish when $x_{1}$ and $x_{2}$ are taken to be $\alpha$ and $\beta$. Such a choice implies that the disturbed orientation of the boundary is no longer parallel to the symmetry plane, and so non-vanishing $\operatorname{det} \hat{\mathbf{W}}$ is in agreement with the general conclusions of $\S 3.2$. The fact that $\operatorname{det} \hat{\mathbf{W}} \neq 0$ ensures that the linear part of Eq. (41), considered as an in- 


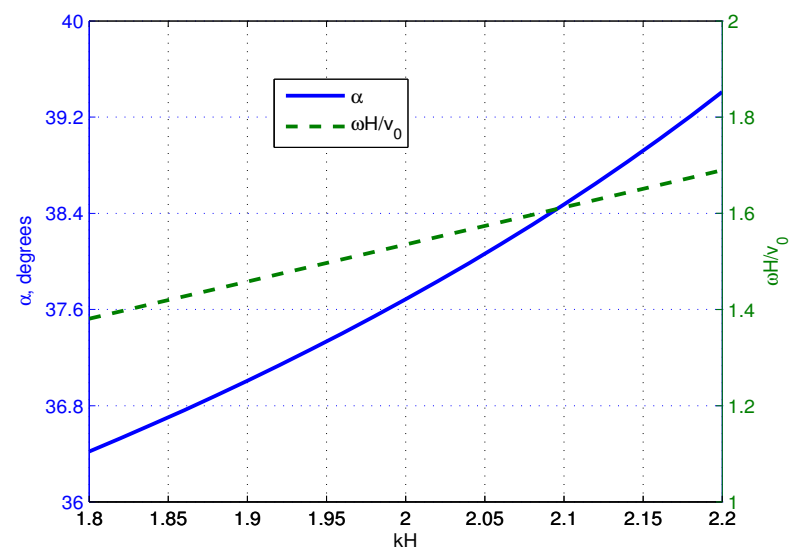

Figure 6: Branch of passband SAWs in GaSb/InAs on surface (001).

homogeneous system of three real linear independent equations in unknown $\omega, x_{1}=\alpha$ and $x_{2}=\beta$ depending on other parameter(s), is always solvable and so the sought branch of passband SAWs does exist. In particular, we can compute the frequency $\omega$ of the passband SAW and the corresponding angles $\alpha, \beta$ as functions of the angle $\gamma$ at a fixed $k$. The dependencies of $\alpha$ and $\beta$ on $\gamma$ for $k H=2$ are shown in Fig. 7. The symmetric and antisymmetric appearance of the curves in Fig. 7 is due to the crystallographic symmetry of layers and to our definition of the orientation angles.

The passband SAW may also exist in phononic crystals formed of elastically isotropic layers (often called isotropic superlattices), where it must be composed by a single sagittally polarized decaying partial mode which produces no traction on the boundary of the structure. The parameters of such SAW must satisfy two equations, as it has been indicated in the end of $\$ 5$ ). Bearing this in mind when searching for the passband SAWs in isotropic su- 


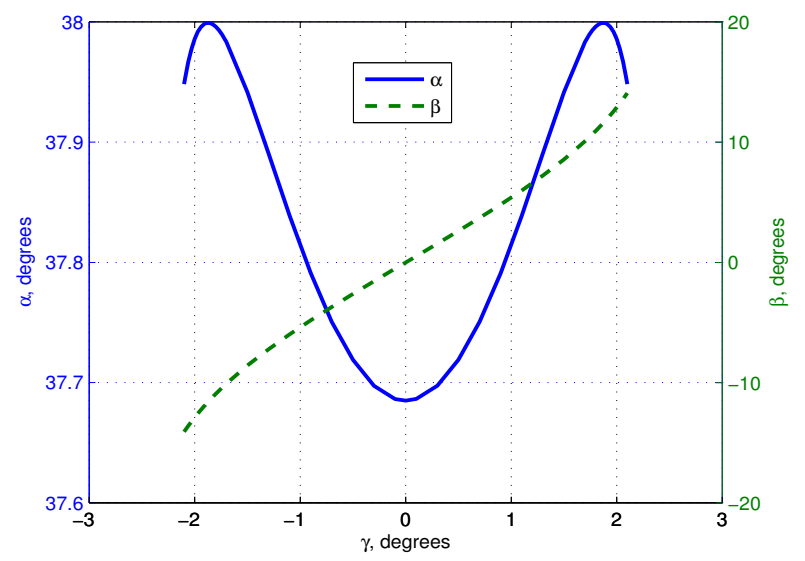

Figure 7: Orientations of the boundary surface of GaSb/InAs and of the propagation direction on it, which admit the passband SAWs in GaSb/InAs at $k H=2$.

perlattices, one can vary, e.g., the thickness of one of the layers and compute the corresponding real values of $\omega$ and $k$. Having not found any publications reporting on passband SAWs in isotropic superlattices, we provide below an example of such SAW in the bilayered phononic crystal composed of isotropic $\mathrm{Al}$ and $\mathrm{W}$ layers, with $\mathrm{Al}$ being the exterior layer.

The spectra of SAWs in the full stopbands and of the leaky waves in the passbands of half-infinite $\mathrm{Al} / \mathrm{W}$ structure were computed in Ref. [14]. For our purpose, we are interested in one of the leaky-wave branches which comes about in the case of identical thicknesses of the $\mathrm{Al}$ and $\mathrm{W}$ layers. For the sake of clarity, a part of this branch showing the dependence of real part of leakywave frequency on $k H$ is depicted in Fig. $8^{2}$, which is in a good agreement

\footnotetext{
${ }^{2}$ Here we use the material constants $c_{11}=11.1 \cdot 10^{10} \mathrm{~N} / \mathrm{m}^{2}, c_{44}=2.5 \cdot 10^{10} \mathrm{~N} / \mathrm{m}^{2}, \rho=$ $2695 \mathrm{~kg} / \mathrm{m}^{3}$ for $\mathrm{Al}$, and $c_{11}=50 \cdot 10^{10} \mathrm{~N} / \mathrm{m}^{2}, c_{44}=15.75 \cdot 10^{10} \mathrm{~N} / \mathrm{m}^{2}, \rho=19250 \mathrm{~kg} / \mathrm{m}^{3}$
} 


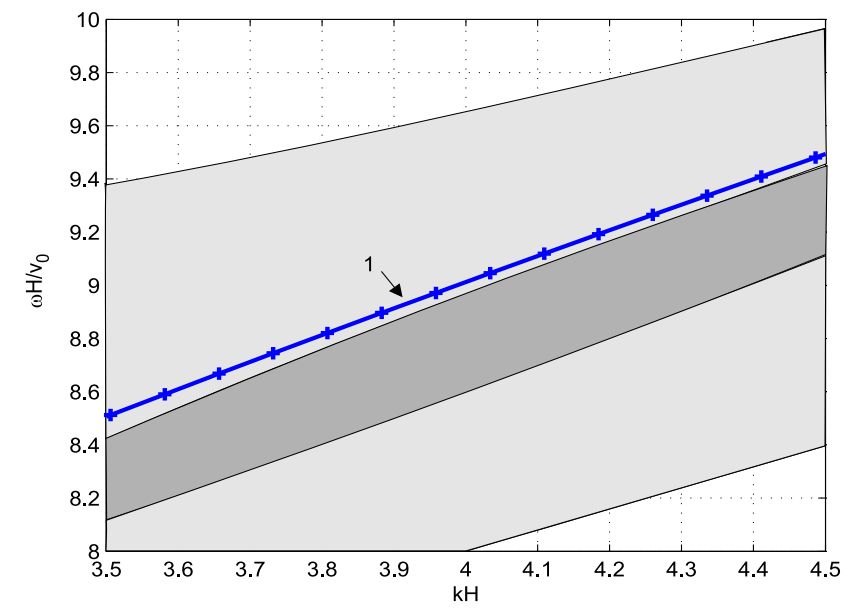

Figure 8: Branch of leaky waves in $\mathrm{Al} / \mathrm{W}$. The layer thicknesses are equal to each other. Curve 1 - real part of the parameter $\omega H / v_{0}$ of leaky waves. Light grey - passbands with one pair of bulk modes. Dark grey - passbands where all four sagittal modes are bulk ones.

with the results of [14] (cf. Fig. 2 in [14]). According to our computations, the imaginary part of the leaky SAW frequency abruptly decreases within a very narrow interval of $k H$-values and vanishes in there, thus revealing the occurrence of the non-leaky passband SAW (Fig. 9). Figure 10 displays the parameters $\omega H / v_{0}$ and $k H$ of the passband SAW as functions of the ratio of $\mathrm{Al}$ layer thickness $h_{A l}$ to $\mathrm{W}$ layer thickness $h_{W}$.

for $\mathrm{W}$. The value of $v_{0}$ is equal to the transverse bulk wave velocity in $\mathrm{Al}$, like in Ref. [14]. 


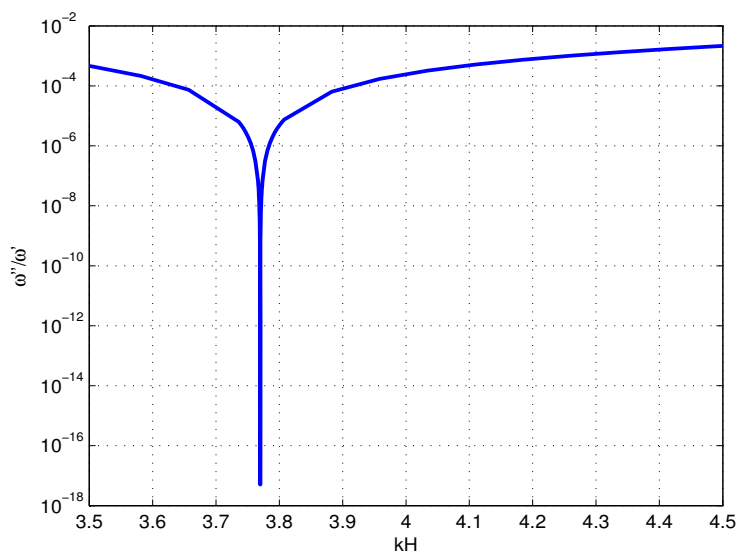

Figure 9: Ratio of the imaginary part $\omega^{\prime \prime}$ of the leaky-wave frequency to its real part $\omega^{\prime}$ versus $k H$ in $\mathrm{Al} / \mathrm{W}$. The layer thicknesses are equal.

\section{Concluding remarks}

In this paper we have investigated possible occurrences of the "true" (nonleaky) SAWs with frequency $\omega$ and tangential wavenumber $k$ falling in the passbands of the Floquet-Bloch spectrum of a 1D phononic crystal viewed as a periodically layered half-space with traction-free boundary. For this purpose, we analyzed how many equations are to be imposed on $\omega, k$ and possibly (usually) some other parameters of the problem, which characterize the propagation geometry and the layer properties, in order for the passband SAW to exist. This number is different for the passbands allowing for either two or four bulk partial modes, and it also depends on whether the crystallographic symmetry is involved. It is shown in the paper that if there is no symmetry plane parallel or orthogonal to the half-space boundary, then the existence of SAWs in the passbands with two or four bulk modes 


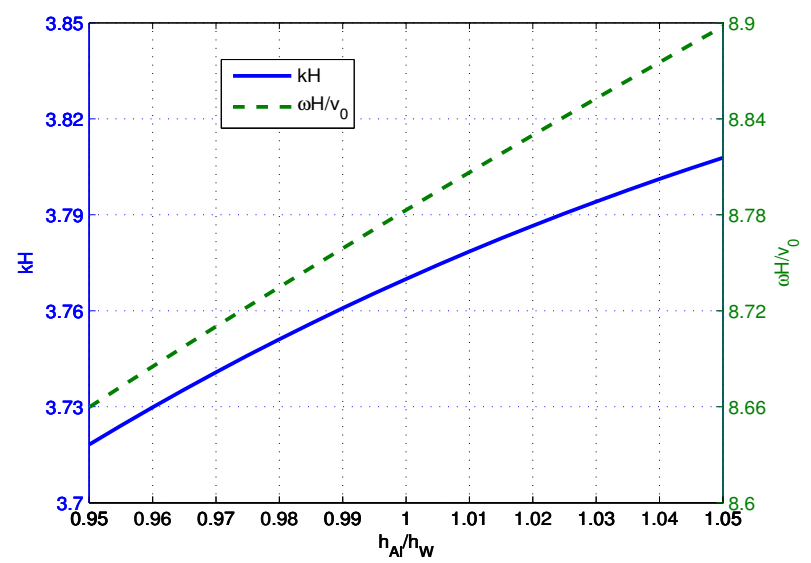

Figure 10: Branch of passband SAWs in AL/W.

is conditioned by three or five real equations, respectively. If all layers of the structure possess a common symmetry plane parallel to the layer interfaces, then the number of conditions required for the existence of SAWs in the passbands with two or four bulk modes reduces to two and three, respectively. The latter conclusion is valid as well in the case where the layers possess a common symmetry plane orthogonal to their interfaces and the SAW propagation direction along the boundary is maintained orthogonal to this symmetry plane. In the case where the sagittal plane is a symmetry plane, the sagittally polarized passband SAW must satisfy three equations. At the same time, if there are two symmetry planes, one parallel to the sagittal plane and the other parallel to the layer interfaces or perpendicular to the direction of propagation, then only two equations are to be fulfilled for the passband SAW to occur. In particular, fulfilling two equations is required for the existence of passband SAWs in the phononic crystals built from elas- 
tically isotropic layers. No SAW can occur in the passbands with four bulk modes, provided that the arrangement of generally anisotropic layers in a period is symmetric with respect to the period midplane.

The existence of SAWs in the passbands is tighter constrained in comparison with the more usual case of their existence in the full stopbands, that is, in the spectral zones where no bulk modes can occur. According to Ref. [26], the dispersion equation for SAWs in a full stopband is equivalent to one real equation which usually relates variations of the frequency $\omega$ and of some other problem parameter, e.g., $k$, without touching other parameters, i.e. keeping all of them fixed. On the contrary, the existence of passband SAWs normally always involve two or more real equations, which therefore necessarily couple variations of $\omega$ and $k$ with that of other problem parameter(s). Nevertheless, the occurrence of passband SAWs is not an extraordinary case. For instance, they exist in the practically important semiconductor superlattices, such as GaAs/AlAs considered in [19] or InAs/GaSb considered in the present work. Note that, on computing the numerical examples of passband SAWs displayed in Figs. 2 and 5-7, we considered variation of $\omega$ as coupled with variations of $k$ and of the orientation angle(s) describing the geometry of the problem. But it is equally possible to keep a suitable geometry fixed and to vary instead the material constants of the constitutive layers. For example, one could start from the passband SAW in the InAs/GaSb superlattice and then gradually change the elastic moduli and the density, pursuing at the same time the branch of SAW solutions and thereby arriving by continuity at a passband SAW in some other superlattice, say, of the same crystallographic symmetry but with desirable material properties. On this ground, it is quite 
feasible to expect existence of passband SAWs in the interrelated family of superlattices made of semiconductors $\mathrm{A}_{3} \mathrm{~B}_{5}$.

\section{Acknowledgments}

The authors thank V.I. Alshits and A.A. Kutsenko for helpful discussions. This work was supported by the Ministry of Science and Higher Education of the Russian Federation within the State assignment FSRC "Crystallography and Photonics" RAS. A. Darinskii also thanks Ministry of Science and Higher Education of the Russian Federation for financial support in the framework of Increase Competitiveness Program of NUST "MISiS' (project No. K22017-079). 


\section{References}

[1] S. Rokhlin, D. Chimenti, P. Nagy, Physical Ultrasonics of Composites, Oxford University Press (2011).

[2] K.-Y. Hashimoto (Ed.), Rf Bulk Acoustic Wave Filters for Communications, Artech House (2009).

[3] A. N. Podlipenets, Surface Love waves in orthotropic regularly layered composites. Mech. Comp. Mater. 18 (1982) 734-737.

[4] R.E. Camley, B. Djafari-Rouhani, L. Dobrzynski, A.A. Maradudin, Transverse elastic waves in periodically layered infinite and semi-infinite media. Phys. Rev. B 27 (1983) 7318-7329.

[5] E. H. El Boudouti, B. Djafari-Rouhani, E. M. Khourdifi, L. Dobrzynski, Surface and interface elastic waves in superlattices: transverse localized and resonant modes, Phys. Rev. B 48 (1993) 10987-10997.

[6] E. H. El Boudouti, B. Djafari-Rouhani, A. Akjouji, L. Dobrzynski, Theory of surface and interface transverse elastic waves in $N$-layer superlattices, Phys. Rev. B 54 (1996) 14728-14741.

[7] S.-Y. Ren and Y.-C. Chang, Theory of confinement effects in finite onedimensional phononic crystals, Phys. Rev. B 75 (2007) 212301.

[8] Ph. Gatignol, C. Potel, J.F. de Belleval, Two families of modal waves for periodic structures with two field functions: a Cayleigh-Hamilton approach, Acustica-Acta Acustica 93 (2007) 959-975. 
[9] A. L. Shuvalov, O. Poncelet, S. V. Golkin, Existence and spectral properties of shear horizontal surface waves in vertically periodic half-spaces. Proc. R. Soc. A 465 (2009) 1489-1511.

[10] M. Alami, E.H. El Boudouti, B. Djafari-Rouhani, Y. El Hassouani, A. Talbi, Surface acoustic waves in one-dimensional piezoelectric-metallic phononic crystal: Effect of a cap layer, Ultrasonics 90 (2018) 80-97.

[11] B. Djafari-Rouhani, L. Dobrzynski, O. Hardouin Duparc, R. E. Camley, A. A. Maradudin, Sagittal elastic waves in periodically layered infinite and semi-infinite media. Phys. Rev. B 28 (1983) 1711-1720.

[12] A. Nougaoui, B. Djafari-Rouhani, Elastic waves in periodically layered infinite and semi-infinite anisotropic media. Surf. Sci. 185 (1987) 125153.

[13] A. N. Podlipenets, N. A. Shul'ga, Numerical study of the propagation of Rayleigh and Lamb waves in orthotropic periodic structures. Int. Appl. Mech. 23 (1987) 1013.

[14] E. H. El Boudouti, B. Djafari-Rouhani, A. Nougaoui, Surface elastic waves in superlattices: Sagittal localized and resonant modes, Phys. Rev. B 51 (1995) 13801-13804.

[15] A. M. B. Braga, G. Herrmann, Floquet waves in anisotropic periodically layered composites. J. Acoust. Soc. Am. 91 (1992) 1211-1227.

[16] A. Nougaoui, B. Djafari Rouhani, Complex band structure of acoustic waves in superlattices. Surf. Sci. 199 (1988) 623-637. 
[17] T. Aono, S. Tamura, Surface and pseudosurface acoustic waves in superlattices, Phys. Rev. B 58 (1998) 4838-4845.

[18] A. Nougaoui, B. Djafari-Rouhani, Vibrations in elastic and piezoelectric superlattices, J. Electron. Spectrosc. Relat. Phenom. 45 (1987) 197-206.

[19] Y. El Hassouani, E.H. El Boudouti, B. Djafari-Rouhani, H. Aynaou, and L. Dobrzynski, Surface and interface acoustic waves in solid-fluid superlattices: Green's function approach, Phys. Rev. B 74 (2006) 144306.

[20] E.H. El Boudouti, B. Djafari-Rouhani, One-Dimensional Phononic Crystals, in Acoustic Metamaterials and Phononic Crystals, Ed.: P.A. Deymier, Springer, Berlin, Heidelberg, 45-94 (2013).

[21] I. Quotane, E.H. El Boudouti, B. Djafari-Rouhani, Y. El Hassouani, V. R. Velasco, Bulk and surface acoustic waves in solid- fluid Fibonacci layered materials, Ultrasonics 61 (2015) 40-51.

[22] E.H. El Boudouti, B. Djafari-Rouhani, A. Akjouji, L. Dobrzynski, Acoustic waves in solids and fluid layered materials. Surf. Sci. Rep. 64 (2009) 471-594.

[23] P.A. Deymier (Ed.), Acoustic Metamaterials and Phononic Crystals, Springer, Berlin, Heidelberg (2013).

[24] V. Laude, Phononic Crystals: Artificial Crystals for Sonic, Acoustic, and Elastic Waves, Series: De Gruyter Studies in Mathematical Physics 26 (2015). 
[25] A. Khelif, A. Adibi (Eds.), Phononic Crystals. Fundamentals and Applications, Springer, Berlin, Heidelberg (2016).

[26] A.N. Darinskii and A.L. Shuvalov, Surface acoustic waves on onedimensional phononic crystals of general anisotropy: Existence considerations, Phys. Rev. B 98 (2018) 024309.

[27] J. Lothe and D. M. Barnett, On the existence of surface wave solutions for anisotropic half-spaces with free surface, J. Appl. Phys. 47 (1976) 428-433.

[28] D. M. Barnett and J. Lothe, Free surface (Rayleigh) waves in anisotropic media: the surface impedance method, Proc. R. Soc. Lond. A402 (1985) 135-152.

[29] G. W. Farnell, "Properties of elastic surface waves," in Physical Acoustics, v. 6, Eds.: W. P. Mason and R. N. Thurston, 1970, pp. 109-166.

[30] V. I. Alshits and J. Lothe, Comments on the relation between surface wave theory and the theory of reflection, Wave Motion 3 (1981) 297-310.

[31] S.A. Gunderson, L. Wang, J. Lothe, Secluded supersonic elastic surface waves. Wave Motion 14 (1991) 129-143.

[32] A.A. Maznev, A.G. Every, Secluded supersonic surface waves in germanium, Phys. Lett. A 197 (1995) 423-427.

[33] A.N. Darinskii, V.I. Alshits, J. Lothe, V.N. Lyubimov, A.L. Shuvalov, An existence criterion for the branch of two-component surface waves in anisotropic elastic media, Wave Motion 28 (1998) 241-257. 
[34] A. N. Stroh, Steady state problems in anisotropic elasticity, J. Math. Phys. 41 (1962) 77 - 103.

[35] M. C. Pease III, Methods of Matrix Algebra, Academic Press, NY, 1965.

[36] A.L. Shuvalov, A sextic formalism for three-dimensional elastodynamics of cylindrically anisotropic radially inhomogeneous materials, Proc. Roy. Soc. Lond. A 459 (2003), 1611-1639.

[37] A. L.Shuvalov, E. Le Clezio, G. Feuillard, The state-vector formalism and the Peano-series solution for modelling guided waves in functionally graded anisotropic piezoelectric plates, Int. J. Eng. Sci. 46(9) (2008) 929-947.

[38] http://www.matprop.ru/ 